\title{
ARL ACADEMIC HeALTH SCIENCES LiBRARY STATISTICS 2009-2010
}

Compiled and Edited by

Martha KyrILLIDOU

SHANEKA MORRIS

GARY ROEBUCK

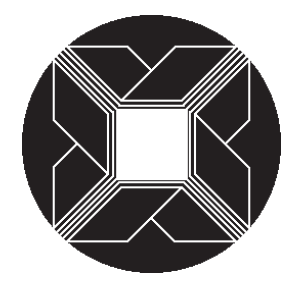

Association of ReSEARCh LibRARIES WASHINGTON, DC 2012 
ARL Academic Health Sciences Library Statistics 2009-2010

The tables presented in this publication are not indicative of performance and outcomes and should not be used as measures of library quality. In comparing any individual library to ARL medians or to other ARL members, one must be careful to make such comparisons within the context of differing institutional and local goals and characteristics.

The ARL Academic Health Sciences Library Statistics datafiles and accompanying documentation are available at www.arl.org/stats/annualsurveys/med/index.shtml.

Published by the Association of Research Libraries

Washington, DC 20036

www.arl.org

ISSN 1538-9006

ISBN 1-59407-879-3 online

EAN 978-159407-879-8 online

(C) 2012

The compilation is copyrighted by the Association of Research Libraries. Blanket permission is granted to reproduce and distribute copies of this work for nonprofit, educational, or library purposes, provided that the author, source, and copyright notice are included on each copy. This permission is in addition to rights of reproduction granted under Sections 107, 108, and other provisions of the US Copyright Act.

The paper used in this publication meets the minimum requirements of the American National Standard for Information Science and National Information Standards Organization standard-Permanence of Paper for Publications and Documents in Libraries and Archives, ANSI/NISO Z39.48-1992(R1997). 


\section{CONTENTS}

Highlights: ARL Academic Health Sciences Library Statistics 2009-2010...................................... 5

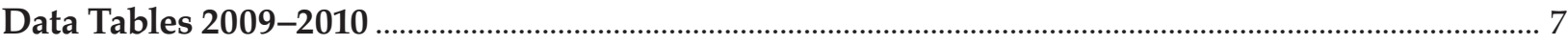

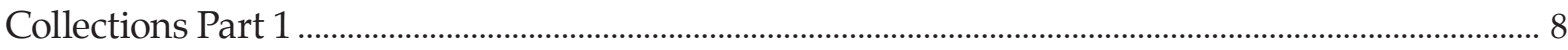

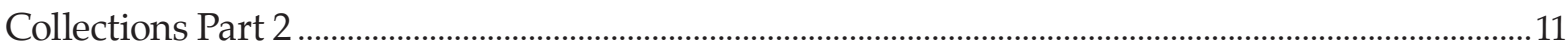

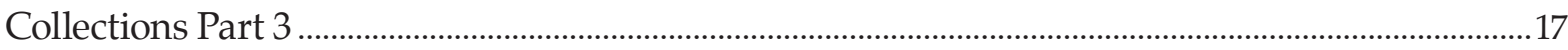

Expenditure

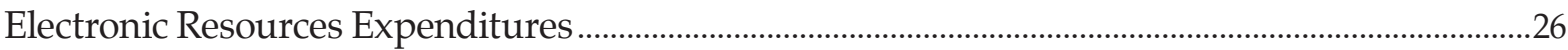

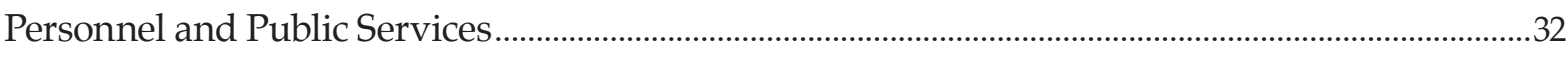

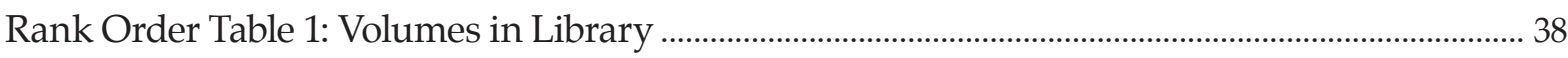

Rank Order Table 2: Volumes Added (Gross) ...............................................................................................39

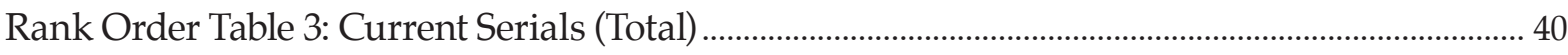

Rank Order Table 4: Total Library Expenditures......................................................................................4

Rank Order Table 5: Total Staff ................................................................................................................. 42

Rank Order Table 6: Total Electronic Resources Expenditures........................................................... 43

ARL Statistics Questionnaire 2009-2010 ....................................................................................................... 44

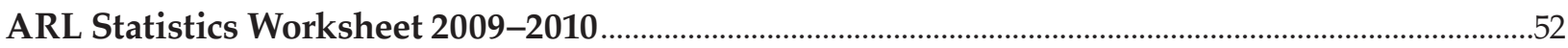

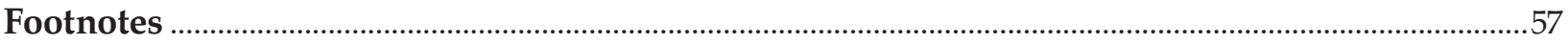

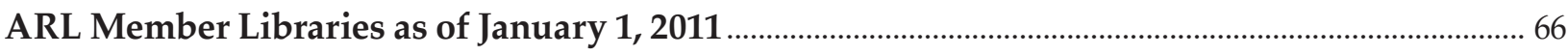





\section{Highlights}

\section{ARL Academic Health Sciences Library Statistics 2009-2010}

- Out of 115 ARL university libraries, 61 responded to this survey. ${ }^{1}$

- Health sciences libraries reported median values of 227,492 volumes held and 1,926 gross volumes added. Also, these libraries employed the full-time equivalent of 2,061 staff members in the fiscal year 2009-2010.

- Responding libraries reported total expenditures of $\$ 229,202,513 .^{2}$ As seen in the graph below, materials expenditures made up the largest portion of the total, with over $50 \%$ of aggregated expenses falling under a materials-related category.

- Health sciences libraries reported a total of $\$ 91,354,237$ in electronic materials expenditures, or a median of almost $89 \%$ of their total materials budgets. This includes a total of $\$ 88,531,989$ in electronic serials expenditures.

\section{Expenditures in ARL Academic Health Sciences Libraries 2009-2010}

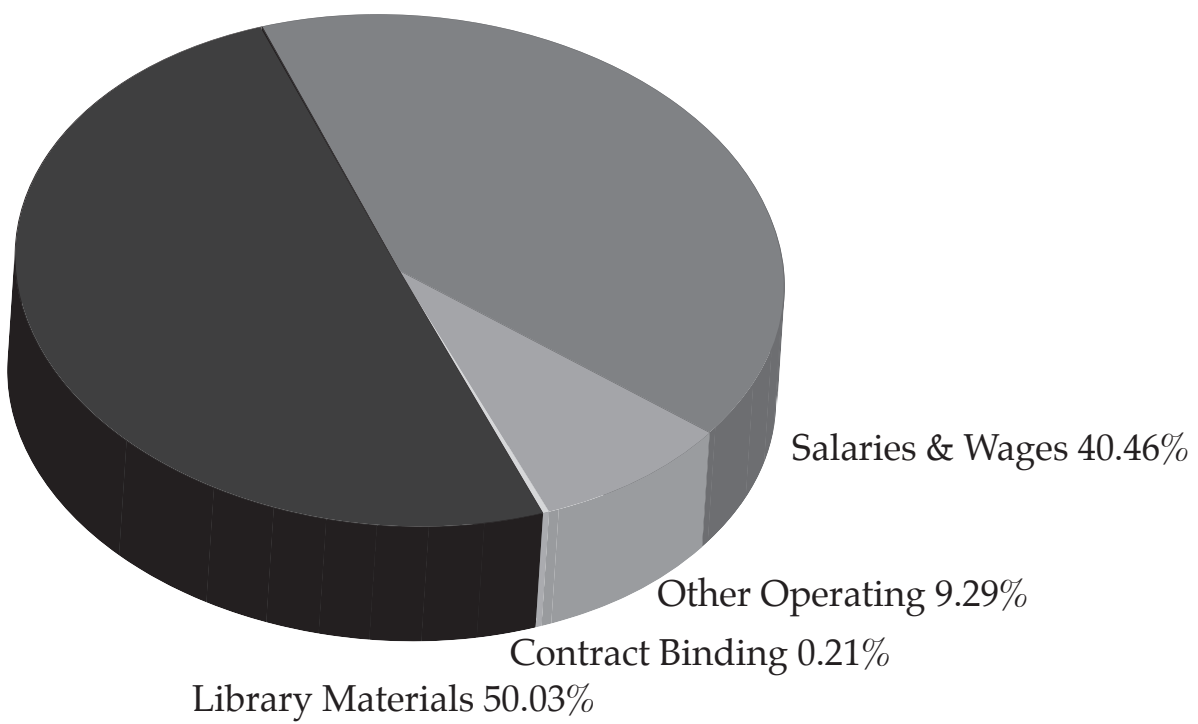

1 Seventy-six ARL university libraries included data for a health sciences library in the 2009-2010 ARL Statistics. Among them, Alberta, British Columbia, Brown, Calgary, Johns Hopkins, Laval, Louisville, McGill, Manitoba, Missouri, Ohio, Ottawa, Pittsburgh, SUNY-Buffalo, Texas A\&M, Toronto, and Wayne State did not complete this survey. McGill completed this survey in the past, but chose not to complete this survey in $2009-2010$. Cornell and Indiana did not include health sciences library data in the ARL Statistics, but did respond to this survey.

2 This figure includes Canadian universities, whose expenditures were converted to US dollars at the rate of 1.0556 Can $\$=1$ US $\$$, the average monthly noon exchange rate published in the Bank of Canada Review for the period July 2009 to June 2010. 

Data Tables

2009-2010 


\section{Collections Part 1}

Notes

Volumes In Volumes Added Library

(Gross)

Volumes Added

(Net)

Titles Held

Monographs

Purchased

(Volumes)

\begin{tabular}{|c|c|c|c|c|c|c|}
\hline ALABAMA & $b+$ & 15,727 & 333 & $-5,595$ & 8,138 & 195 \\
\hline CALIFORNIA, DAVIS & + & 376,666 & 3,714 & 2,448 & 376,666 & 1,153 \\
\hline CALIFORNIA, LOS ANGELES & $b+$ & 675,011 & 5,776 & 5,256 & . & 652 \\
\hline CALIFORNIA, SAN DIEGO & + & 244,052 & 5,136 & 3,334 & . & 1,828 \\
\hline CASE WESTERN RESERVE & + & 424,850 & 1,143 & 1,143 & 119,113 & 630 \\
\hline CONNECTICUT & $b$ & 199,975 & 2,631 & 1,490 & 51,571 & 1,305 \\
\hline CORNELL & $\mathrm{G}+$ & 204,256 & 988 & 229 & 72,463 & 313 \\
\hline DARTMOUTH & $b+$ & 255,150 & 964 & $-5,392$ & 73,122 & 669 \\
\hline DUKE & bG & 248,383 & 1,525 & 719 & . & 207 \\
\hline EMORY & G+ & 227,492 & 2,319 & $-14,244$ & 68,645 & 1,007 \\
\hline FLORIDA & G+ & 318,235 & 500 & $-1,132$ & . & 242 \\
\hline HAWAII & & 35,293 & 1,650 & 1,527 & 12,182 & 6,903 \\
\hline HOWARD & $b$ & 135,211 & 368 & 190 & 136,718 & 1,245 \\
\hline ILLINOIS, CHICAGO & + & 753,782 & 19,507 & 10,487 & 348,295 & 10,278 \\
\hline INDIANA & $b$ & 206,879 & 2,527 & -814 & 98,504 & 677 \\
\hline IOWA & $\mathrm{G}+$ & 413,364 & 61,962 & 53,880 & 153,648 & 721 \\
\hline KANSAS & $\mathrm{G}+$ & 185,320 & 10,918 & 9,950 & 85,421 & 410 \\
\hline KENTUCKY & + & 245,895 & 4,322 & 2,409 & 98,133 & 1,360 \\
\hline LOUISIANA STATE & G+ & 49,366 & 277 & 276 & . & 110 \\
\hline MCMASTER & + & 144,354 & 2,790 & -383 & 62,726 & 2,318 \\
\hline
\end{tabular}

8 - ARL Academic Health Sciences Library Statistics 2009-2010 


\section{Collections Part 1}

Notes

Volumes In Volumes Added Library

(Gross)

Volumes Added

(Net)

Titles Held

Monographs

Purchased

(Volumes)

\begin{tabular}{|c|c|c|c|c|c|c|}
\hline MIAMI & + & 205,060 & 532 & $-2,785$ & 67,805 & 221 \\
\hline MICHIGAN & b & 607,251 & 44,915 & $-26,191$ & 322,825 & 2,533 \\
\hline MONTREAL & + & 325,025 & 6,312 & $-10,570$ & 143,102 & 6,484 \\
\hline NEW YORK & $b+$ & 232,415 & 6,477 & 1,843 & 57,105 & 1,039 \\
\hline NORTH CAROLINA & G+ & 349,881 & 3,167 & 2,856 & 140,790 & 1,124 \\
\hline NORTHWESTERN & + & 250,556 & 1,931 & $-41,909$ & & 912 \\
\hline PENNSYLVANIA & G & 205,913 & 1,524 & 1,518 & & . \\
\hline PENNSYLVANIA STATE & $b G+$ & 45,905 & 331 & 184 & 17,286 & 235 \\
\hline QUEEN'S & G+ & 135,415 & 1,364 & $-6,994$ & . & 1,079 \\
\hline ROCHESTER & $b+$ & 273,609 & 2,713 & 2,660 & & 1,301 \\
\hline SASKATCHEWAN & G+ & 165,026 & 1,926 & 1,006 & 50,740 & 1,069 \\
\hline SOUTH CAROLINA & G & 115,412 & 574 & $-1,706$ & 33,751 & 152 \\
\hline TENNESSEE & $b+$ & 193,772 & 1,518 & $-27,451$ & 56,931 & 233 \\
\hline TEXAS TECH & $b G+$ & 319,452 & 5,464 & 5,218 & 5,218 & 2,817 \\
\hline TULANE & G+ & 156,789 & 618 & $-2,199$ & . & 618 \\
\hline UTAH & $\mathrm{b}$ & 195,139 & 459 & $-2,502$ & 74,942 & 336 \\
\hline VANDERBILT & + & 191,793 & 1,907 & $-4,108$ & 73,541 & 786 \\
\hline VIRGINIA & G+ & 113,905 & 1,070 & $-11,020$ & . & 2,297 \\
\hline WASHINGTON & + & 287,234 & 2,030 & 2,030 & . & 159 \\
\hline WASHINGTON U.-ST. LOUIS & $b+$ & 310,935 & 16,133 & 15,960 & 112,500 & 1,246 \\
\hline WISCONSIN & $\mathrm{b}$ & 315,009 & 1,575 & $-3,115$ & 162,756 & 383 \\
\hline
\end{tabular}




\section{Collections Part 1}

\begin{tabular}{|c|c|c|c|c|c|c|}
\hline & Notes & $\begin{array}{r}\text { Volumes In } \\
\text { Library } \\
1\end{array}$ & $\begin{array}{r}\text { Volumes Added } \\
\text { (Gross) } \\
1 \mathrm{~b} . \mathrm{i}\end{array}$ & $\begin{array}{r}\text { Volumes Added } \\
\text { (Net) } \\
1 \mathrm{~b}\end{array}$ & $\begin{array}{r}\text { Titles Held } \\
2\end{array}$ & $\begin{array}{r}\text { Monographs } \\
\text { Purchased } \\
\text { (Volumes) } \\
3\end{array}$ \\
\hline YALE & + & 508,064 & 19,766 & 19,764 & 299,929 & 920 \\
\hline
\end{tabular}

\section{Summary Data}

$\begin{array}{rr}\text { Volumes In Library } & \text { Volumes Added } \\ 1 & \text { (Gross) }\end{array}$
Volumes Added

(Net)

$1 \mathrm{~b}$
Titles Held

2
Monographs

Purchased

(Volumes)

\begin{tabular}{lrrrr}
\hline Mean & 265,806 & 5,162 & -85 & 115,145 \\
Median & 227,492 & 1,926 & 276 & 74,242 \\
High & 866,448 & 61,962 & 53,880 & 394,648 \\
Low & 12,506 & 216 & $-41,909$ & 596 \\
Totals & $16,214,163$ & $-5,194$ & 5,077 \\
Number of Libraries & 61 & 614,872 & 61 & 48,278 \\
Reporting & & 61 & 82,170
\end{tabular}




\section{Collections Part 2}

Notes Current Serials

Total
5
Current Serials

Purchased
Current Serials

Purchased Electronic
Current Serials

Purchased Print 5a.ii

\begin{tabular}{|c|c|c|c|c|c|}
\hline ALABAMA & $b+$ & 4,131 & 218 & 132 & 86 \\
\hline ARIZONA & $b+$ & 12,124 & 8,949 & 8,806 & 143 \\
\hline BOSTON & $\mathrm{bG}$ & 4,818 & 4,175 & 4,152 & 23 \\
\hline CALIFORNIA, DAVIS & + & 2,352 & 1,923 & 0 & 1,923 \\
\hline CALIFORNIA, IRVINE & $b+$ & 9,388 & 6,247 & 5,869 & 378 \\
\hline CALIFORNIA, LOS ANGELES & $b+$ & 0 & 0 & . & . \\
\hline CALIFORNIA, SAN DIEGO & + & 7,782 & 5,987 & 5,550 & 437 \\
\hline CASE WESTERN RESERVE & + & 15,380 & 15,380 & 15,299 & 81 \\
\hline CHICAGO & + & 31,427 & 28,927 & 28,065 & 862 \\
\hline CINCINNATI & $b+$ & 0 & 0 & . & . \\
\hline COLUMBIA & $\mathrm{G}+$ & 0 & 0 & 0 & 0 \\
\hline CONNECTICUT & $\mathrm{b}$ & 18,045 & 1,765 & 1,162 & 603 \\
\hline CORNELL & $\mathrm{G}+$ & 9,600 & . & . & . \\
\hline DARTMOUTH & $b+$ & 1,660 & 1,362 & 1,260 & 102 \\
\hline DUKE & $\mathrm{bG}$ & 1,019 & 1,019 & 925 & 94 \\
\hline EMORY & $\mathrm{G}+$ & 9,132 & 2,083 & 2,002 & 81 \\
\hline FLORIDA & $\mathrm{G}+$ & 393 & 393 & . & 393 \\
\hline FLORIDA STATE & $\mathrm{bG}+$ & 734 & 734 & 725 & 9 \\
\hline GEORGE WASHINGTON & $\mathrm{bG}+$ & 2,726 & 1,015 & 987 & 28 \\
\hline GEORGETOWN & $\mathrm{bG}+$ & 4,211 & 3,261 & 3,249 & 12 \\
\hline HARVARD & $\mathrm{b}$ & 520 & 268 & 0 & 268 \\
\hline HAWAII & & 5,233 & 1,306 & 1,275 & 31 \\
\hline HOWARD & $\mathrm{b}$ & 6,982 & 1,212 & 642 & 570 \\
\hline ILLINOIS, CHICAGO & + & 766 & 766 & . & 766 \\
\hline INDIANA & $\mathrm{b}$ & 8,088 & 1,435 & 1,413 & 22 \\
\hline IOWA & $\mathrm{G}+$ & 7,511 & 4,991 & 4,861 & 130 \\
\hline KANSAS & $\mathrm{G}+$ & 957 & 937 & 827 & 110 \\
\hline KENTUCKY & + & 223 & 213 & . & 213 \\
\hline LOUISIANA STATE & $\mathrm{G}+$ & 4,510 & 4,500 & 4,470 & 30 \\
\hline MCMASTER & + & 4,599 & 4,056 & 3,916 & 140 \\
\hline
\end{tabular}




\section{Collections Part 2}

Notes Current Serials

Total
Current Serials
Purchased
Current Serials

Purchased

Electronic
Current Serials

Purchased Print

5a.ii

\begin{tabular}{|c|c|c|c|c|c|}
\hline MIAMI & + & 11,358 & 11,319 & 11,277 & 42 \\
\hline MONTREAL & + & 3,674 & 3,634 & 3,329 & 305 \\
\hline NEW YORK & $b+$ & . & . & . & \\
\hline NORTH CAROLINA & G+ & 6,766 & 5,611 & 5,295 & 316 \\
\hline NORTHWESTERN & + & 9,738 & 9,738 & 9,738 & \\
\hline OHIO STATE & $b+$ & 6,327 & 3,037 & 2,946 & 91 \\
\hline PENNSYLVANIA & G & 4,088 & 4,088 & 4,023 & 65 \\
\hline PENNSYLVANIA STATE & $b G+$ & 8,820 & 6,810 & 6,800 & 10 \\
\hline QUEEN'S & $\mathrm{G}+$ & . & . & . & \\
\hline ROCHESTER & $b+$ & 0 & . & . & . \\
\hline SASKATCHEWAN & G+ & 5,656 & 4,960 & 4,871 & 89 \\
\hline SOUTH CAROLINA & G & 29,728 & 1,204 & 1,204 & 0 \\
\hline TENNESSEE & $b+$ & 1,247 & 808 & 662 & 146 \\
\hline TEXAS TECH & $b G+$ & 25,380 & 23,106 & 22,804 & 302 \\
\hline TULANE & G+ & 8,669 & 2,761 & 2,661 & 100 \\
\hline UTAH & $\mathrm{b}$ & 3,488 & 1,533 & 1,420 & 113 \\
\hline VANDERBILT & + & 5,143 & 3,729 & 3,709 & 20 \\
\hline VIRGINIA & G+ & 5,073 & 2,568 & 2,356 & 212 \\
\hline WASHINGTON & + & 0 & 0 & . & . \\
\hline WASHINGTON U.-ST. LOUIS & $b+$ & 4,190 & 3,290 & 3,260 & 30 \\
\hline WISCONSIN & $\mathrm{b}$ & 4,740 & 1,108 & 1,024 & 84 \\
\hline
\end{tabular}

12 - ARL Academic Health Sciences Library Statistics 2009-2010 


\section{Collections Part 2}

Notes $\quad$ Current Serials
Total
5
Current Serials
Purchased

Current Serials

Purchased

Electronic

Current Serials

Purchased Print

5a.i

\begin{tabular}{lccc}
\hline YALE & $+10,193$ & 7,568 \\
& G - Government documents not included in serials counts & \\
+ - See Footnotes & b - Basis of volume count is bibliographic
\end{tabular}

\section{Summary Data}

Current Serials Total

5
Current Serials

Purchased
Current Serials

Purchased Electronic

5a.i
Current Serials Purchased Print

5a.ii

$\begin{array}{lrrrr}\text { Mean } & 7,461 & 4,870 & 5,168 & 3,249 \\ \text { Median } & 5,143 & 2,806 & 109 \\ \text { High } & 31,427 & 30,027 & 29,848 & 1,923 \\ \text { Low } & 223 & 213 & 132 \\ \text { Totals } & 380,511 & 243,478 & 232,577 \\ \text { Number of Libraries Reporting } & 51 & 50 & 45\end{array}$




\section{Collections Part 2} Consortial
Current Serials
Not Purchased Freely Accessible
Current Serials

Not Purchased

Print
Current Serials

Not Purchased GovDocs 5b.iv

\begin{tabular}{|c|c|c|c|c|c|c|}
\hline ALABAMA & $b+$ & 3,913 & . & 3,912 & 1 & . \\
\hline ARIZONA & $b+$ & 3,175 & 0 & 3,133 & 42 & 0 \\
\hline BOSTON & $\mathrm{bG}$ & 643 & 0 & 625 & 0 & 18 \\
\hline CALIFORNIA, DAVIS & + & 429 & 0 & 0 & 429 & 0 \\
\hline CALIFORNIA, IRVINE & $b+$ & 3,141 & 0 & 1,293 & 15 & 1,833 \\
\hline CALIFORNIA, LOS ANGELES & $b+$ & 0 & . & . & . & . \\
\hline CALIFORNIA, SAN DIEGO & + & 1,795 & . & 1,761 & 17 & 17 \\
\hline CASE WESTERN RESERVE & + & 0 & 0 & 0 & 0 & 0 \\
\hline CHICAGO & + & 2,500 & 0 & 2,500 & 0 & . \\
\hline CINCINNATI & $b+$ & 0 & . & . & . & . \\
\hline COLUMBIA & $\mathrm{G}+$ & 0 & . & . & . & . \\
\hline CONNECTICUT & $\mathrm{b}$ & 16,280 & 15,780 & 500 & 0 & 0 \\
\hline CORNELL & $\mathrm{G}+$ & . & . & . & . & . \\
\hline DARTMOUTH & $b+$ & 298 & . & . & 155 & 143 \\
\hline DUKE & $\mathrm{bG}$ & 0 & . & . & . & . \\
\hline EMORY & $\mathrm{G}+$ & 7,049 & . & 7,049 & . & . \\
\hline FLORIDA & $\mathrm{G}+$ & 0 & . & . & . & . \\
\hline FLORIDA STATE & $\mathrm{bG}+$ & . & . & . & . & . \\
\hline GEORGE WASHINGTON & bG+ & 1,711 & 0 & 1,706 & 5 & 0 \\
\hline GEORGETOWN & bG+ & 950 & . & . & . & . \\
\hline HARVARD & $\mathrm{b}$ & 252 & 0 & 252 & 0 & 0 \\
\hline HAWAII & & 3,927 & 0 & 3,895 & 32 & . \\
\hline HOWARD & $b$ & 5,770 & 221 & 5,549 & 0 & 0 \\
\hline ILLINOIS, CHICAGO & + & 0 & . & . & . & . \\
\hline INDIANA & $\mathrm{b}$ & 6,653 & 390 & 6,250 & 13 & 0 \\
\hline IOWA & $\mathrm{G}+$ & 2,520 & 0 & 2,486 & 34 & 0 \\
\hline KANSAS & $\mathrm{G}+$ & 20 & 0 & 20 & 0 & 0 \\
\hline KENTUCKY & + & 10 & . & . & 10 & 0 \\
\hline LOUISIANA STATE & $\mathrm{G+}$ & 10 & 0 & 0 & 10 & 0 \\
\hline MCMASTER & + & 543 & 0 & 526 & 17 & 0 \\
\hline
\end{tabular}

14 - ARL Academic Health Sciences Library Statistics 2009-2010 


\section{Collections Part 2}

Current Serials

Not Purchased

Consortial
Current Serials

Not Purchased

Freely Accessible

$5 b . i i$
Current Serials

Not Purchased

Print

5b.iii
Current Serials

Not Purchased GovDocs

\begin{tabular}{|c|c|c|c|c|c|c|}
\hline MIAMI & + & 39 & 0 & 0 & 39 & 0 \\
\hline MICHIGAN & $\mathrm{b}$ & 446 & 0 & 371 & 72 & 3 \\
\hline MINNESOTA & $b+$ & . & . & . & . & . \\
\hline MONTREAL & + & 40 & 0 & 0 & 40 & 0 \\
\hline NEW MEXICO & + & 62 & 0 & 0 & 62 & 0 \\
\hline NEW YORK & $b+$ & . & . & . & . & . \\
\hline NORTH CAROLINA & G+ & 1,155 & 0 & 1,114 & 41 & 0 \\
\hline NORTHWESTERN & + & . & . & . & . & . \\
\hline OHIO STATE & $b+$ & 3,290 & 2,887 & 105 & 298 & 0 \\
\hline OKLAHOMA & + & 0 & 0 & 0 & 0 & 0 \\
\hline OKLAHOMA STATE & + & . & . & . & . & . \\
\hline PENNSYLVANIA & G & 0 & . & . & . & . \\
\hline PENNSYLVANIA STATE & bG+ & 2,010 & 0 & 2,010 & 0 & . \\
\hline QUEEN'S & G+ & . & . & . & . & . \\
\hline ROCHESTER & $b+$ & . & . & . & . & . \\
\hline SASKATCHEWAN & G+ & 696 & 0 & 680 & 16 & . \\
\hline SOUTH CAROLINA & G & 28,524 & 27,465 & 1,059 & 0 & 0 \\
\hline SOUTHERN CALIFORNIA & + & 943 & 0 & 943 & 0 & 0 \\
\hline SOUTHERN ILLINOIS & G & 0 & 0 & 0 & 0 & 0 \\
\hline SUNY-STONY BROOK & bG & 4,745 & 1,545 & 3,198 & 2 & 0 \\
\hline TEMPLE & $b+$ & 2,242 & 0 & 2,242 & 0 & 0 \\
\hline TENNESSEE & $b+$ & 439 & 214 & 201 & 3 & 21 \\
\hline TEXAS TECH & bG+ & 2,274 & 0 & 2,253 & 21 & 0 \\
\hline TULANE & G+ & 5,908 & 3,075 & 2,833 & 0 & 0 \\
\hline UTAH & $\mathrm{b}$ & 1,955 & 1,713 & 224 & 10 & 8 \\
\hline VANDERBILT & + & 1,414 & 579 & 831 & 0 & 4 \\
\hline VIRGINIA & G+ & 2,505 & . & 1,694 & 811 & . \\
\hline WASHINGTON & + & 0 & . & . & . & . \\
\hline WASHINGTON U.-ST. LOUIS & $b+$ & 900 & 360 & 540 & . & . \\
\hline WISCONSIN & $\mathrm{b}$ & 3,632 & 0 & 3,624 & 8 & 0 \\
\hline
\end{tabular}




\section{Collections Part 2}

\begin{tabular}{|c|c|c|c|c|c|c|}
\hline & Notes & $\begin{array}{r}\text { Current Serials } \\
\text { Not Purchased } \\
5 b\end{array}$ & $\begin{array}{r}\text { Current Serials } \\
\text { Not Purchased } \\
\text { Consortial } \\
5 \mathrm{~b} . \mathrm{i}\end{array}$ & $\begin{array}{r}\text { Current Serials } \\
\text { Not Purchased } \\
\text { Freely } \\
\text { Accessible } \\
5 \mathrm{~b} . \mathrm{ii}\end{array}$ & $\begin{array}{r}\text { Current Serials } \\
\text { Not Purchased } \\
\text { Print } \\
5 b . i i i\end{array}$ & $\begin{array}{r}\text { Current Serials } \\
\text { Not Purchased } \\
\text { GovDocs } \\
5 \mathrm{~b} . \mathrm{iv}\end{array}$ \\
\hline YALE & + & 2,625 & 0 & 2,625 & 0 & 0 \\
\hline
\end{tabular}

\section{Summary Data}

\begin{tabular}{|c|c|c|c|c|c|}
\hline & $\begin{array}{r}\text { Current Serials Not } \\
\text { Purchased } \\
5 \mathrm{~b}\end{array}$ & $\begin{array}{r}\text { Current Serials Not } \\
\text { Purchased } \\
\text { Consortial } \\
5 \mathrm{~b} . \mathrm{i}\end{array}$ & $\begin{array}{r}\text { Current Serials Not } \\
\text { Purchased Freely } \\
\text { Accessible } \\
5 \mathrm{~b} . \mathrm{ii}\end{array}$ & $\begin{array}{r}\text { Current Serials Not } \\
\text { Purchased Print } \\
5 b . \text {.iii }\end{array}$ & $\begin{array}{r}\text { Current Serials Not } \\
\text { Purchased GovDocs } \\
5 \mathrm{~b} \text {.iv }\end{array}$ \\
\hline Mean & 3,034 & 4,930 & 2,000 & 85 & 256 \\
\hline Median & 1,875 & 1,545 & 1,700 & 19 & 18 \\
\hline Low & 10 & 214 & 20 & 1 & 3 \\
\hline Totals & 127,433 & 54,229 & 68,004 & 2,203 & 2,047 \\
\hline $\begin{array}{l}\text { Number of Libraries } \\
\text { Reporting }\end{array}$ & 42 & 11 & 34 & 26 & 8 \\
\hline
\end{tabular}




\section{Collections Part 3}

\begin{tabular}{|c|c|c|c|c|c|c|c|c|}
\hline Notes & $\begin{array}{r}\text { Microform } \\
\text { Units } \\
7\end{array}$ & $\begin{array}{c}\text { Government } \\
\text { Documents }\end{array}$ & $\begin{array}{r}\text { Computer } \\
\text { Files } \\
9\end{array}$ & $\begin{array}{r}\text { Manuscripts } \\
\text { and Archives } \\
10\end{array}$ & $\begin{array}{r}\text { Cartographic } \\
\text { Materials } \\
11\end{array}$ & $\begin{array}{r}\text { Graphic } \\
\text { Materials } \\
12\end{array}$ & $\begin{array}{r}\text { Audio } \\
\text { Materials } \\
13\end{array}$ & $\begin{array}{l}\text { deo } \\
14\end{array}$ \\
\hline
\end{tabular}

\begin{tabular}{|c|c|c|c|c|c|c|c|c|c|}
\hline ALABAMA & $b+$ & 0 & 0 & 124 & 0 & 0 & 1,193 & 505 & 361 \\
\hline ARIZONA & $b+$ & 4,700 & 0 & 0 & 15 & 34 & 20,000 & 100 & 4,375 \\
\hline BOSTON & bG & 29 & 0 & 365 & 751 & 0 & 0 & 17 & 112 \\
\hline CALIFORNIA, DAVIS & + & 12,769 & 0 & 345 & 0 & 0 & 481 & 1,091 & 267 \\
\hline CALIFORNIA, IRVINE & $b+$ & 278,696 & 17,912 & 1,317 & 552 & 1,377 & 8,599 & 2,426 & 1,961 \\
\hline CALIFORNIA, LOS ANGELES & $b+$ & 64,853 & 5,891 & 2,241 & 1,578 & 3 & 43,992 & 3,573 & 1,655 \\
\hline CALIFORNIA, SAN DIEGO & + & 14,466 & 0 & 1,092 & 0 & 0 & 2,895 & 2,888 & 9 \\
\hline CASE WESTERN RESERVE & + & 3,752 & 0 & 500 & 1,000 & 0 & 51,833 & 1,300 & 1,321 \\
\hline CHICAGO & + & 29,562 & 0 & 1,843 & 0 & 0 & 0 & 0 & 488 \\
\hline CINCINNATI & $b+$ & 0 & 0 & 0 & 3,070 & 0 & 6,000 & 2,140 & 851 \\
\hline COLUMBIA & G+ & 52,822 & . & 957 & 4,475 & . & 13,474 & . & 1,112 \\
\hline CONNECTICUT & $\mathrm{b}$ & 0 & 0 & 475 & 0 & 0 & 0 & 38 & 1,116 \\
\hline CORNELL & $\mathrm{G}+$ & . & 0 & 612 & 0 & . & . & 29 & 971 \\
\hline DARTMOUTH & $b+$ & 13,059 & 0 & 565 & 0 & 3 & 20,313 & 5,835 & 1,478 \\
\hline DUKE & bG & 193 & 0 & 30,992 & 12,464 & 93 & 591,609 & 1,269 & 3,338 \\
\hline EMORY & G+ & 15 & 0 & 240 & 257 & . & - & 4,318 & 1,476 \\
\hline FLORIDA & $\mathrm{G}+$ & 1,747 & . & 66 & . & . & . & . & -303 \\
\hline FLORIDA STATE & bG+ & 0 & 0 & 0 & 0 & 0 & 0 & 0 & 107 \\
\hline GEORGE WASHINGTON & $b G+$ & 0 & 0 & 380 & 60 & 0 & 3,660 & 994 & 1,194 \\
\hline GEORGETOWN & bG+ & 0 & 0 & 61 & 0 & 0 & 69 & 0 & 100 \\
\hline HARVARD & $\mathrm{b}$ & . & . & . & . & . & . & . & . \\
\hline HAWAII & & 0 & . & 120 & 0 & 0 & 0 & 0 & 25 \\
\hline HOWARD & $\mathrm{b}$ & 20,000 & 0 & 0 & 0 & 0 & 0 & 65 & 1,520 \\
\hline ILLINOIS, CHICAGO & + & 61,616 & 0 & 773 & . & . & . & 965 & 2,393 \\
\hline INDIANA & $\mathrm{b}$ & 747 & 0 & 537 & 185 & 0 & 0 & 200 & 182 \\
\hline IOWA & $\mathrm{G}^{+}$ & . & . & . & . & . & . & 211 & 969 \\
\hline KANSAS & $\mathrm{G}+$ & 94 & 0 & 1,518 & 0 & 0 & 153 & 62 & 1,281 \\
\hline KENTUCKY & + & 2 & 0 & 14 & 0 & 0 & 31,598 & 1,282 & 1,407 \\
\hline LOUISIANA STATE & $\mathrm{G}+$ & 0 & 0 & 353 & 0 & 0 & 0 & 0 & 716 \\
\hline MCMASTER & + & 5,241 & 0 & 51 & 855 & 10 & 0 & 650 & 716 \\
\hline
\end{tabular}




\section{Collections Part 3}

$\begin{array}{rrrrrrrr}\text { Notes } & \text { Microform } & \text { Government } & \text { Computer } & \text { Manuscripts } & \text { Cartographic } & \text { Graphic } & \text { Audio } \\ \text { Units } & \text { Documents } & \text { Files } & \text { and Archives } & \text { Materials } & \text { Materials } & \text { Materials } & \text { Video } \\ 7 & 8 & 9 & 10 & 11 & 12 & 13\end{array}$

\begin{tabular}{|c|c|c|c|c|c|c|c|c|c|}
\hline MIAMI & + & 2,837 & 0 & . & 610 & . & . & . & 1,753 \\
\hline MICHIGAN & $\mathrm{b}$ & 0 & 0 & 795 & 3 & 0 & 0 & 2 & 25 \\
\hline MINNESOTA & $b+$ & 67,186 & 19,979 & 645 & 80 & 7 & . & . & . \\
\hline MONTREAL & + & 31,540 & 0 & 1,240 & 0 & 56 & 12,623 & 998 & 1,780 \\
\hline NEW MEXICO & + & 1 & . & 464 & 1,925 & 0 & 7,428 & 1,357 & 2,123 \\
\hline NEW YORK & $b+$ & 4 & . & 122 & 707 & . & 8,861 & 52 & 208 \\
\hline NORTH CAROLINA & $\mathrm{G+}$ & 18,911 & 0 & 26 & 0 & 31 & 16,057 & 30 & 1,128 \\
\hline NORTHWESTERN & + & 0 & 0 & 714 & . & 0 & . & 478 & 1,148 \\
\hline OHIO STATE & $b+$ & 115 & 0 & 544 & 1,150 & 1,669 & 10,000 & 81 & 1,077 \\
\hline OKLAHOMA & + & 650 & 0 & 154 & 6,207 & 0 & 0 & 0 & 6,579 \\
\hline OKLAHOMA STATE & + & 44,260 & 0 & 16 & 168 & 2 & 3,852 & 3,433 & 1,586 \\
\hline PENNSYLVANIA & G & . & . & . & . & . & . & . & . \\
\hline PENNSYLVANIA STATE & bG+ & 0 & 0 & 4 & 1,129 & . & . & 3 & 371 \\
\hline QUEEN'S & G+ & . & . & . & . & . & . & . & . \\
\hline ROCHESTER & $b+$ & 0 & 0 & 23 & 1,500 & 0 & 5,000 & 65 & 77 \\
\hline SASKATCHEWAN & G+ & 2,477 & 0 & 46 & 0 & 0 & 10,986 & 169 & 49 \\
\hline SOUTH CAROLINA & G & 4,721 & 0 & 8 & 0 & 0 & 0 & 81 & 1,415 \\
\hline SOUTHERN CALIFORNIA & + & 8,286 & 0 & 707 & 35 & 0 & 12,591 & 2,357 & 652 \\
\hline SOUTHERN ILLINOIS & G & 6,094 & 0 & 60 & 417 & 0 & 0 & 2,574 & 3,426 \\
\hline SUNY-STONY BROOK & bG & 0 & 0 & 0 & 0 & 0 & 0 & 0 & 0 \\
\hline TEMPLE & $b+$ & 0 & 0 & 2 & 0 & 0 & 0 & 0 & 2,702 \\
\hline TENNESSEE & $b+$ & 328 & 0 & 339 & 205 & 0 & 0 & 8 & 733 \\
\hline TEXAS TECH & bG+ & 2,550 & 0 & 2,570 & 750 & 0 & 786 & 5,190 & 7,520 \\
\hline TULANE & G+ & 0 & 0 & 324 & 1,351 & 0 & 0 & 0 & 0 \\
\hline UTAH & $\mathrm{b}$ & 15,273 & 0 & 876 & 0 & 0 & 1,073 & 0 & 898 \\
\hline VANDERBILT & + & 9,119 & 0 & 1,116 & 3,973 & 2 & 0 & 1,272 & 1,352 \\
\hline VIRGINIA & G+ & 1 & 0 & 280 & 932 & 0 & 0 & 0 & 1,875 \\
\hline WASHINGTON & + & 86,413 & 0 & 1,001 & 0 & 0 & 453 & 0 & 106 \\
\hline WASHINGTON U.-ST. LOUIS & $b+$ & 1,783 & . & 1,375 & 3,031 & . & . & 2 & 484 \\
\hline WISCONSIN & $\mathrm{b}$ & 6,667 & 0 & 95 & 3,670 & 0 & 11 & 740 & 1,377 \\
\hline
\end{tabular}

18 - ARL Academic Health Sciences Library Statistics 2009-2010 


\section{Collections Part 3}

$\begin{array}{rrr}\text { Notes } & \text { Microform } & \text { Government } \\ \text { Units } & \text { Documents } \\ 7 & 8\end{array}$
Computer
Files

Manuscripts

and Archives
Cartographic

Materials
Graphic

Materials

Materials
Audio
Materials

Materials
13
Film and Video

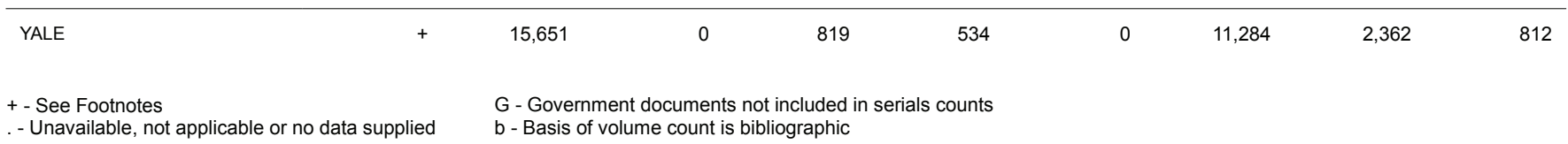

\section{Summary Data}

\begin{tabular}{|c|c|c|c|c|c|c|c|}
\hline Microform & Government & Computer & Manuscripts & Cartographic & Graphic & Audio & Film and \\
\hline Units & Documents & Files & and Archives & Materials & Materials & Materials & Video \\
\hline
\end{tabular}

\begin{tabular}{|c|c|c|c|c|c|c|c|c|}
\hline Mean & 21,689 & 14,594 & 1,175 & 1,676 & 274 & 30,927 & 1,219 & 1,317 \\
\hline Median & 5,241 & 17,912 & 464 & 803 & 21 & 8,599 & 695 & 1,112 \\
\hline High & 278,696 & 19,979 & 30,992 & 12,464 & 1,669 & 591,609 & 5,835 & 7,520 \\
\hline Low & 1 & 5,891 & 2 & 3 & 2 & 11 & 2 & -303 \\
\hline Totals & 889,230 & 43,782 & 59,906 & 53,639 & 3,287 & 896,874 & 51,212 & 72,454 \\
\hline $\begin{array}{l}\text { Number of Libraries } \\
\text { Reporting }\end{array}$ & 41 & 3 & 51 & 32 & 12 & 29 & 42 & 55 \\
\hline
\end{tabular}




\section{EXPENDITURES}

\begin{tabular}{|c|c|c|c|c|c|c|}
\hline Notes & $\begin{array}{r}\text { Monographs } \\
16 a\end{array}$ & $\begin{array}{r}\text { Current } \\
\text { Serials } \\
16 \mathrm{~b}\end{array}$ & $\begin{array}{r}\text { Other Library } \\
\text { Materials } \\
16 \mathrm{c}\end{array}$ & $\begin{array}{r}\text { Miscellaneous } \\
\text { Materials } \\
16 \mathrm{~d}\end{array}$ & $\begin{array}{r}\text { Total Library } \\
\text { Materials } \\
16\end{array}$ & $\begin{array}{r}\text { Contract } \\
\text { Binding } \\
17\end{array}$ \\
\hline
\end{tabular}

\begin{tabular}{|c|c|c|c|c|c|c|c|}
\hline ALABAMA & $b+$ & 1,508 & 99,678 & 24,489 & 6,279 & 131,954 & 1,439 \\
\hline ARIZONA & $b+$ & 19,136 & $1,217,403$ & 161,251 & 0 & $1,397,790$ & 0 \\
\hline CALIFORNIA, DAVIS & + & 44,807 & $1,309,719$ & . & . & $1,354,526$ & . \\
\hline CALIFORNIA, LOS ANGELES & $b+$ & 264,655 & $1,600,363$ & . & . & $1,865,018$ & 42,268 \\
\hline CALIFORNIA, SAN DIEGO & + & 205,039 & $1,750,511$ & 12,450 & 166,053 & $2,134,053$ & \\
\hline CASE WESTERN RESERVE & + & 170,191 & $1,465,072$ & 0 & 0 & $1,635,263$ & 2,030 \\
\hline CONNECTICUT & b & 206,899 & $1,611,921$ & 41,369 & 328,348 & $2,188,537$ & 9,797 \\
\hline CORNELL & G+ & 111,081 & $1,303,965$ & 772 & 2,223 & $1,418,041$ & 0 \\
\hline DARTMOUTH & $b+$ & 41,174 & $1,092,325$ & 12 & 0 & $1,133,511$ & 7,301 \\
\hline DUKE & bG & 47,281 & $1,433,376$ & 55,862 & 222,657 & $1,759,176$ & 2,012 \\
\hline EMORY & G+ & 149,324 & $2,043,368$ & 10,600 & 325,884 & $2,529,176$ & 15,911 \\
\hline FLORIDA & G+ & 7,741 & $1,872,227$ & 3,019 & 5,243 & $1,888,230$ & \\
\hline HAWAII & & 118,270 & 317,282 & 0 & 0 & 435,552 & . \\
\hline HOWARD & $b$ & 42,000 & $1,055,555$ & 110,174 & 0 & $1,207,729$ & 6,000 \\
\hline ILLINOIS, CHICAGO & + & 212,328 & $1,742,020$ & 290,841 & 0 & $2,245,189$ & 22,656 \\
\hline INDIANA & $b$ & 112,876 & $1,489,624$ & 157,973 & 86,544 & $1,847,017$ & 70 \\
\hline IOWA & G+ & 126,042 & $2,948,496$ & 1,606 & 49,429 & $3,125,573$ & . \\
\hline KANSAS & G+ & 28,339 & $1,430,268$ & 41,831 & 0 & $1,500,438$ & 14,000 \\
\hline KENTUCKY & + & 144,909 & $1,981,712$ & 4,466 & 37,995 & $2,169,082$ & 15,654 \\
\hline LOUISIANA STATE & $\mathrm{G}+$ & 7,338 & 378,053 & 0 & 0 & 385,391 & 1,177 \\
\hline MCMASTER & + & 366,616 & $1,169,613$ & 262,447 & 43,978 & $1,842,654$ & 746 \\
\hline
\end{tabular}




\section{EXPENDITURES}

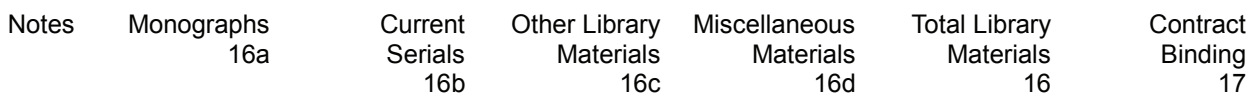

\begin{tabular}{|c|c|c|c|c|c|c|c|}
\hline MIAMI & + & 108,953 & $1,853,415$ & 0 & 0 & $1,962,368$ & 0 \\
\hline MICHIGAN & $b$ & 105,426 & $1,507,759$ & . & 2,445 & $1,615,630$ & 0 \\
\hline MONTREAL & + & 247,615 & $1,757,839$ & 0 & 0 & $2,005,454$ & 6,065 \\
\hline NEW YORK & $b+$ & 102,327 & $1,836,746$ & 336,412 & . & $2,275,485$ & . \\
\hline NORTH CAROLINA & G+ & 211,517 & $2,405,166$ & 555,913 & 0 & $3,172,596$ & 12,888 \\
\hline NORTHWESTERN & + & 54,713 & $1,613,909$ & 410,247 & 3,828 & $2,082,697$ & 7,954 \\
\hline PENNSYLVANIA & G & 137,176 & $2,385,579$ & . & . & $2,522,755$ & . \\
\hline PENNSYLVANIA STATE & bG+ & 29,333 & $1,180,395$ & 142,126 & 45,136 & $1,396,990$ & 0 \\
\hline QUEEN'S & G+ & 120,887 & $1,537,565$ & 0 & 0 & $1,658,452$ & 3,629 \\
\hline ROCHESTER & $b+$ & 125,260 & $1,867,300$ & 0 & 0 & $1,992,560$ & 18,327 \\
\hline SASKATCHEWAN & G+ & 483,433 & $2,004,581$ & . & . & $2,488,014$ & 5,141 \\
\hline SOUTH CAROLINA & G & 15,735 & 564,701 & 93,893 & 1,695 & 676,024 & 0 \\
\hline TENNESSEE & $b+$ & 78,607 & $1,493,978$ & 12,003 & 25,183 & $1,609,771$ & 462 \\
\hline TEXAS TECH & bG+ & 312,120 & $2,133,580$ & 54,826 & 516,284 & $3,016,810$ & 24,427 \\
\hline TULANE & G+ & 84,031 & $1,233,675$ & 120,659 & 0 & $1,438,365$ & 0 \\
\hline UTAH & $\mathrm{b}$ & 27,626 & $1,098,175$ & 14,352 & 5,694 & $1,145,847$ & 0 \\
\hline VANDERBILT & + & 296,778 & $2,171,816$ & 213,517 & 39,800 & $2,721,911$ & 0 \\
\hline VIRGINIA & G+ & 164,342 & $1,710,650$ & 7,210 & 6,911 & $1,889,113$ & 0 \\
\hline WASHINGTON & + & 124,871 & $2,637,610$ & 28 & 3,749 & $2,766,258$ & 35 \\
\hline WASHINGTON U.-ST. LOUIS & $b+$ & 268,070 & $2,568,954$ & 508,975 & 114,246 & $3,460,245$ & 27,892 \\
\hline WISCONSIN & $\mathrm{b}$ & 16,952 & $1,366,225$ & 2,742 & 67,079 & $1,452,998$ & 32,484 \\
\hline
\end{tabular}




\section{EXPENDITURES}

\begin{tabular}{|c|c|c|c|c|c|c|c|}
\hline & Notes & $\begin{array}{r}\text { Monographs } \\
16 a\end{array}$ & $\begin{array}{r}\text { Current } \\
\text { Serials } \\
16 b\end{array}$ & $\begin{array}{r}\text { Other Library } \\
\text { Materials } \\
16 c\end{array}$ & $\begin{array}{r}\text { Miscellaneous } \\
\text { Materials } \\
16 \mathrm{~d}\end{array}$ & $\begin{array}{r}\text { Total Library } \\
\text { Materials } \\
16\end{array}$ & $\begin{array}{r}\text { Contract } \\
\text { Binding } \\
17\end{array}$ \\
\hline YALE & + & 752,352 & $1,637,311$ & 11,144 & 0 & $2,400,807$ & 5,470 \\
\hline $\begin{array}{l}\text { - See Footnotes } \\
\text { - Unavailable, not applicable or no data supplied }\end{array}$ & & sis of volume co & ibliographic & t & & & \\
\hline
\end{tabular}

\section{Summary Data}

$\begin{array}{rrrrrr}\text { Monographs } & \text { Current Serials } & \text { Other Library } & \text { Miscellaneous } & \text { Total Library } & \text { Contract Binding } \\ 16 \mathrm{a} & 16 \mathrm{~b} & \text { Materials } & \text { Materials } & \text { Materials } & 17\end{array}$

\begin{tabular}{|c|c|c|c|c|c|c|}
\hline Mean & 146,571 & $1,519,614$ & 203,728 & 108,220 & $1,879,927$ & 12,503 \\
\hline Median & 111,081 & $1,537,565$ & 87,333 & 39,747 & $1,847,017$ & 6,267 \\
\hline High & 752,352 & $2,948,496$ & $3,692,727$ & 527,096 & $4,223,445$ & 42,268 \\
\hline Low & 1,508 & 99,678 & 12 & 275 & 131,954 & 35 \\
\hline Totals & $8,940,858$ & $92,696,428$ & $9,575,233$ & $3,463,055$ & $114,675,574$ & 487,599 \\
\hline $\begin{array}{l}\text { Number of Libraries } \\
\text { Reporting }\end{array}$ & 61 & 61 & 47 & 32 & 61 & 39 \\
\hline
\end{tabular}




\section{EXPENDITURES}

Notes

Salaries \&
Wages
Professional
Staff
$18 a$

Salaries \&
Wages
Support Staff
$18 b$

Salaries \&
Wages
Student
Assistants

Total Salaries

and Wages

Other
Operating

18 Expenditures

Total Library

Expenditures

\begin{tabular}{|c|c|c|c|c|c|c|c|}
\hline ALABAMA & $b+$ & 88,137 & 66,908 & 10,824 & 165,869 & 11,222 & 310,484 \\
\hline ARIZONA & $b+$ & $1,012,926$ & 484,523 & 56,024 & $1,553,473$ & 334,609 & $3,285,872$ \\
\hline BOSTON & bG & 676,593 & 326,102 & 44,203 & $1,046,898$ & 74,278 & $2,450,360$ \\
\hline CALIFORNIA, DAVIS & + & 453,023 & 481,334 & 47,898 & 982,255 & 91,052 & $2,427,833$ \\
\hline CALIFORNIA, IRVINE & $b+$ & 480,780 & 523,240 & 70,097 & $1,074,117$ & 79,643 & $2,134,491$ \\
\hline CALIFORNIA, LOS ANGELES & $b+$ & $1,027,387$ & 562,797 & 244,569 & $1,834,753$ & 602,329 & $4,344,368$ \\
\hline CALIFORNIA, SAN DIEGO & + & 651,798 & 633,772 & 106,582 & $1,392,152$ & 44,541 & $3,570,746$ \\
\hline CASE WESTERN RESERVE & + & 497,076 & 368,630 & 151,538 & $1,017,244$ & 121,520 & $2,776,057$ \\
\hline CHICAGO & + & $\S 317,883$ & $\S 348,096$ & $\S 72,200$ & $\S 738,179$ & 27,873 & $5,023,063$ \\
\hline CINCINNATI & $b+$ & 875,478 & 109,523 & 65,130 & $1,050,131$ & 553,466 & $3,413,822$ \\
\hline COLUMBIA & G+ & 850,150 & 405,074 & 0 & $1,255,224$ & 126,857 & $3,932,360$ \\
\hline CONNECTICUT & b & $1,177,718$ & 702,613 & 49,721 & $1,930,052$ & 93,677 & $4,222,063$ \\
\hline CORNELL & $\mathrm{G}+$ & 861,462 & 801,961 & 0 & $1,663,423$ & 242,057 & $3,323,521$ \\
\hline DARTMOUTH & $b+$ & 530,272 & 455,355 & 26,276 & $1,011,903$ & 128,987 & $2,281,702$ \\
\hline DUKE & bG & $1,199,822$ & 482,047 & 0 & $1,681,869$ & 333,668 & $3,776,725$ \\
\hline EMORY & $\mathrm{G}+$ & 891,976 & 586,780 & 0 & $1,478,756$ & 243,282 & $4,267,125$ \\
\hline FLORIDA & G+ & 697,693 & 776,011 & 70,554 & $1,544,258$ & 257,462 & $3,689,950$ \\
\hline FLORIDA STATE & bG+ & 269,821 & 69,417 & 28,915 & 368,153 & 30,703 & $1,299,592$ \\
\hline GEORGE WASHINGTON & bG+ & 941,706 & 674,954 & 189,138 & $1,805,798$ & 210,307 & $4,240,503$ \\
\hline GEORGETOWN & bG+ & 707,155 & 308,196 & 36,151 & $1,051,502$ & 209,380 & $3,096,650$ \\
\hline HARVARD & b & $4,261,757$ & $1,083,336$ & 45,006 & $5,390,099$ & $4,203,018$ & $12,179,443$ \\
\hline HAWAII & & 205,617 & 128,837 & . & 334,454 & 54,345 & 824,351 \\
\hline HOWARD & $\mathrm{b}$ & 470,699 & 303,140 & 125,000 & 898,839 & 12,000 & $2,124,568$ \\
\hline ILLINOIS, CHICAGO & + & 752,284 & $1,017,229$ & 81,575 & $1,851,088$ & 358,799 & $4,477,732$ \\
\hline INDIANA & $\mathrm{b}$ & 789,173 & 801,905 & 44,087 & $1,635,165$ & 94,212 & $3,576,464$ \\
\hline IOWA & G+ & 502,168 & 488,671 & 144,734 & $1,135,573$ & 0 & $4,261,146$ \\
\hline KANSAS & G+ & 623,563 & 707,293 & 50,179 & $1,381,035$ & 158,481 & $3,053,954$ \\
\hline KENTUCKY & + & 744,336 & 140,329 & 114,615 & 999,280 & 91,061 & $3,275,077$ \\
\hline LOUISIANA STATE & G+ & 45,740 & 111,160 & 10,857 & 167,757 & 13,308 & 567,633 \\
\hline MCMASTER & + & 537,216 & 762,079 & 73,605 & $1,372,900$ & 90,948 & $3,307,249$ \\
\hline
\end{tabular}




\section{EXPENDITURES}

\begin{tabular}{|c|c|c|c|c|c|c|c|}
\hline & Notes & $\begin{array}{r}\text { Salaries \& } \\
\text { Wages } \\
\text { Professional } \\
\text { Staff } \\
18 a\end{array}$ & $\begin{array}{r}\text { Salaries \& } \\
\text { Wages } \\
\text { Support Staff } \\
18 b\end{array}$ & $\begin{array}{r}\text { Salaries \& } \\
\text { Wages } \\
\text { Student } \\
\text { Assistants } \\
18 \mathrm{c}\end{array}$ & $\begin{array}{r}\text { Total Salaries } \\
\text { and Wages } \\
18\end{array}$ & $\begin{array}{r}\text { Other } \\
\text { Operating } \\
\text { Expenditures } \\
20\end{array}$ & $\begin{array}{r}\text { Total Library } \\
\text { Expenditures } \\
21\end{array}$ \\
\hline MIAMI & + & 927,702 & 722,638 & 0 & $1,650,340$ & 225,953 & $3,838,661$ \\
\hline MICHIGAN & $b$ & 861,010 & 678,923 & 159,073 & $1,699,006$ & 0 & $3,314,636$ \\
\hline MINNESOTA & $b+$ & 946,064 & $1,042,205$ & 205,123 & $2,193,392$ & 809,684 & $6,534,185$ \\
\hline MONTREAL & + & 697,071 & $1,007,194$ & 0 & $1,704,265$ & 18,823 & $3,734,607$ \\
\hline NEW MEXICO & + & $1,125,494$ & 738,828 & 30,000 & $1,894,322$ & 215,219 & $3,167,828$ \\
\hline NEW YORK & $b+$ & $2,017,002$ & $1,210,017$ & 57,621 & $3,284,640$ & $1,045,811$ & $6,605,936$ \\
\hline NORTH CAROLINA & $\mathrm{G}+$ & $\S 2,268,096$ & $\S 1,370,665$ & $\S 143,618$ & $\S 3,782,379$ & 465,804 & $7,433,667$ \\
\hline NORTHWESTERN & + & $1,128,675$ & 387,728 & 0 & $1,516,403$ & 85,208 & $3,692,262$ \\
\hline OHIO STATE & $b+$ & $2,558,669$ & 534,093 & 203,506 & $3,296,268$ & 367,959 & $5,248,453$ \\
\hline OKLAHOMA & + & 431,060 & 778,957 & 118,778 & $1,328,795$ & 198,129 & $3,565,747$ \\
\hline OKLAHOMA STATE & + & $\S 204,772$ & $\S 318,364$ & $\S 52,013$ & $\S 575,149$ & 46,644 & $1,020,134$ \\
\hline PENNSYLVANIA & G & 578,086 & 507,523 & 162,973 & $1,248,582$ & 307,161 & $4,078,498$ \\
\hline PENNSYLVANIA STATE & bG+ & 381,382 & 319,651 & 48,498 & 749,531 & 136,895 & $2,283,416$ \\
\hline QUEEN'S & G+ & 675,452 & 446,924 & 31,983 & $1,154,359$ & 162,392 & $2,978,832$ \\
\hline ROCHESTER & $b+$ & $1,216,407$ & 206,656 & 54,310 & $1,477,373$ & 280,322 & $3,768,582$ \\
\hline SASKATCHEWAN & G+ & 536,667 & 251,977 & 29,784 & 818,428 & . & $3,311,584$ \\
\hline SOUTH CAROLINA & G & 449,599 & 212,458 & 19,665 & 681,722 & 189,807 & $1,547,553$ \\
\hline SOUTHERN CALIFORNIA & + & $1,018,615$ & $1,011,245$ & 139,187 & $2,169,047$ & 409,720 & $5,681,704$ \\
\hline SOUTHERN ILLINOIS & G & 406,670 & 473,405 & 18,302 & 898,377 & 100,725 & $1,763,562$ \\
\hline SUNY-STONY BROOK & bG & $1,305,900$ & 254,694 & 130,352 & $1,690,946$ & 451,879 & $3,695,794$ \\
\hline TEMPLE & $b+$ & 693,117 & 350,331 & 73,351 & $1,116,799$ & 301,180 & $3,844,455$ \\
\hline TENNESSEE & $b+$ & 679,969 & 432,191 & 0 & $1,112,160$ & 40,063 & $2,762,456$ \\
\hline TEXAS TECH & bG+ & $1,175,971$ & 760,525 & 243,765 & $2,180,261$ & 345,449 & $5,566,947$ \\
\hline TULANE & G+ & 396,200 & 192,446 & 13,713 & 602,359 & 36,420 & $2,077,144$ \\
\hline UTAH & $b$ & 808,041 & 725,132 & 138,516 & $1,671,689$ & $1,244,266$ & $4,061,802$ \\
\hline VANDERBILT & + & $1,157,872$ & 551,861 & 11,812 & $1,721,545$ & 708,506 & $5,151,962$ \\
\hline VIRGINIA & G+ & 955,215 & 873,030 & 3,779 & $1,832,024$ & 275,017 & $3,996,154$ \\
\hline WASHINGTON & + & $1,826,826$ & 566,075 & 115,350 & $2,508,251$ & $2,399,765$ & $7,674,309$ \\
\hline WASHINGTON U.-ST. LOUIS & $b+$ & $\S 2,005,940$ & $\S 659,769$ & . & $\S 2,665,709$ & 468,260 & $6,622,106$ \\
\hline WISCONSIN & $b$ & $\S 946,990$ & $\S 293,476$ & $\S 94,576$ & $\S 1,335,042$ & 848,179 & $3,668,703$ \\
\hline
\end{tabular}




\section{EXPENDITURES}

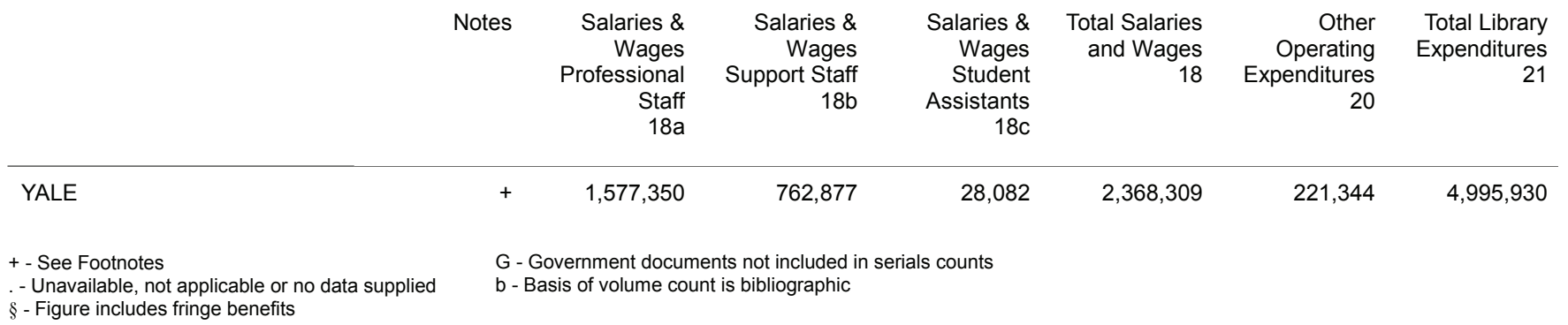

\section{Summary Data}

\begin{tabular}{|c|c|c|c|c|c|c|}
\hline & $\begin{array}{r}\text { Salaries \& } \\
\text { Wages } \\
\text { Professional } \\
\text { Staff } \\
18 a\end{array}$ & $\begin{array}{r}\text { Salaries \& } \\
\text { Wages Support } \\
\text { Staff } \\
18 b\end{array}$ & $\begin{array}{r}\text { Salaries \& } \\
\text { Wages Student } \\
\text { Assistants } \\
18 \mathrm{c}\end{array}$ & $\begin{array}{r}\text { Total Salaries } \\
\text { and Wages } \\
18\end{array}$ & $\begin{array}{r}\text { Other Operating } \\
\text { Expenditures } \\
20\end{array}$ & $\begin{array}{r}\text { Total Library } \\
\text { Expenditures } \\
21\end{array}$ \\
\hline Mean & 903,267 & 546,773 & 84,063 & $1,520,322$ & 367,236 & $3,757,418$ \\
\hline Median & 752,284 & 507,523 & 65,130 & $1,392,152$ & 209,844 & $3,668,703$ \\
\hline Low & 45,740 & 66,908 & 3,779 & 165,869 & 11,222 & 310,484 \\
\hline Totals & $55,099,293$ & $33,353,171$ & $4,287,207$ & $92,739,671$ & $21,299,670$ & $229,202,513$ \\
\hline $\begin{array}{l}\text { Number of Libraries } \\
\text { Reporting }\end{array}$ & 61 & 61 & 51 & 61 & 58 & 61 \\
\hline
\end{tabular}




\section{Electronic Resources Expenditures}

Notes $\begin{array}{r}\text { One-time } \\ \text { Electronic } \\ \text { Resource } \\ \text { Purchases } \\ 22\end{array}$

$\begin{array}{rr}\text { Ongoing } & \text { Total Electronic } \\ \text { Electronic } & \text { Resource } \\ \text { Resource } & \text { Purchases } \\ \text { Purchases } & 22+23\end{array}$

Total Library

Materials

Expenditures

$22+23$

22

23

\begin{tabular}{|c|c|c|c|c|c|c|}
\hline ALABAMA & $b+$ & 0 & 83,285 & 83,285 & 131,954 & 63.12 \\
\hline ARIZONA & $b+$ & 9,937 & $1,314,714$ & $1,324,651$ & $1,397,790$ & 94.77 \\
\hline BOSTON & bG & 33,380 & $1,139,008$ & $1,172,388$ & $1,329,184$ & 88.20 \\
\hline CALIFORNIA, DAVIS & + & 0 & $1,041,423$ & $1,041,423$ & $1,354,526$ & 76.88 \\
\hline CALIFORNIA, IRVINE & $b+$ & 22,869 & 597,835 & 620,704 & 977,214 & 63.52 \\
\hline CALIFORNIA, LOS ANGELES & $b+$ & . & . & . & $1,865,018$ & . \\
\hline CALIFORNIA, SAN DIEGO & + & 84,657 & $1,372,089$ & $1,456,746$ & $2,134,053$ & 68.26 \\
\hline CASE WESTERN RESERVE & + & 3,047 & $1,240,619$ & $1,243,666$ & $1,635,263$ & 76.05 \\
\hline CHICAGO & + & 128,881 & $3,563,846$ & $3,692,727$ & $4,223,445$ & 87.43 \\
\hline CINCINNATI & $b+$ & 111,377 & $1,268,834$ & $1,380,211$ & $1,808,845$ & 76.30 \\
\hline COLUMBIA & G+ & 0 & $1,959,627$ & $1,959,627$ & $2,539,661$ & 77.16 \\
\hline CONNECTICUT & $b$ & 16,323 & $2,012,069$ & $2,028,392$ & $2,188,537$ & 92.68 \\
\hline CORNELL & G+ & 136,477 & $1,153,546$ & $1,290,023$ & $1,418,041$ & 90.97 \\
\hline DARTMOUTH & $b+$ & . & $1,047,086$ & $1,047,086$ & $1,133,511$ & 92.38 \\
\hline DUKE & bG & 2,588 & $1,149,591$ & $1,152,179$ & $1,759,176$ & 65.50 \\
\hline EMORY & G+ & 85,028 & $1,954,297$ & $2,039,325$ & $2,529,176$ & 80.63 \\
\hline FLORIDA & G+ & 1,119 & $1,575,866$ & $1,576,985$ & $1,888,230$ & 83.52 \\
\hline FLORIDA STATE & bG+ & 10,790 & 888,468 & 899,258 & 900,736 & 99.84 \\
\hline GEORGE WASHINGTON & bG+ & 81,831 & $2,042,089$ & $2,123,920$ & $2,224,398$ & 95.48 \\
\hline GEORGETOWN & $b G+$ & 0 & $1,740,431$ & $1,740,431$ & $1,835,470$ & 94.82 \\
\hline HARVARD & $\mathrm{b}$ & 95,026 & $2,290,067$ & $2,385,093$ & $2,544,637$ & 93.73 \\
\hline HAWAII & & 0 & 428,382 & 428,382 & 435,552 & 98.35 \\
\hline HOWARD & $\mathrm{b}$ & 0 & $1,119,796$ & $1,119,796$ & $1,207,729$ & 92.72 \\
\hline ILLINOIS, CHICAGO & + & 15,457 & $1,661,580$ & $1,677,037$ & $2,245,189$ & 74.69 \\
\hline INDIANA & $\mathrm{b}$ & 52,051 & $1,731,522$ & $1,783,573$ & $1,847,017$ & 96.57 \\
\hline IOWA & G+ & 667 & $2,858,237$ & $2,858,904$ & $3,125,573$ & 91.47 \\
\hline KANSAS & G+ & 0 & $1,253,000$ & $1,253,000$ & $1,500,438$ & 83.51 \\
\hline KENTUCKY & + & 37,476 & $1,676,219$ & $1,713,695$ & $2,169,082$ & 79.01 \\
\hline LOUISIANA STATE & G+ & 0 & 371,915 & 371,915 & 385,391 & 96.50 \\
\hline MCMASTER & + & 44,505 & $1,379,224$ & $1,423,730$ & $1,842,654$ & 77.27 \\
\hline
\end{tabular}

26 - ARL Academic Health Sciences Library Statistics 2009-2010 


\section{Electronic Resources Expenditures}

Notes $\begin{array}{r}\text { One-time } \\ \text { Electronic } \\ \text { Resource } \\ \text { Purchases } \\ 22\end{array}$

$\begin{array}{rr}\text { Ongoing } & \text { Total Electronic } \\ \text { Electronic } & \text { Resource } \\ \text { Resource } & \text { Purchases } \\ \text { Purchases } & 22+23\end{array}$

Total Library

Materials

Expenditures
Electronic Resources as a $\%$ of Library Materials Budget

\begin{tabular}{|c|c|c|c|c|c|c|}
\hline MIAMI & + & 23,855 & $1,729,931$ & $1,753,786$ & $1,962,368$ & 89.37 \\
\hline MICHIGAN & $b$ & 40,374 & 674,837 & 715,211 & $1,615,630$ & 44.27 \\
\hline MINNESOTA & $b+$ & 276,901 & $2,282,887$ & $2,559,788$ & $3,495,454$ & 73.23 \\
\hline MONTREAL & + & 169,505 & $1,680,148$ & $1,849,653$ & $2,005,454$ & 92.23 \\
\hline NEW MEXICO & + & 59,901 & 941,651 & $1,001,552$ & $1,057,258$ & 94.73 \\
\hline NEW YORK & $b+$ & . & $1,904,881$ & $1,904,881$ & $2,275,485$ & 83.71 \\
\hline NORTH CAROLINA & G+ & . & . & . & $3,172,596$ & . \\
\hline NORTHWESTERN & + & 11,370 & $1,954,921$ & $1,966,291$ & $2,082,697$ & 94.41 \\
\hline OHIO STATE & $b+$ & 45,626 & $1,249,340$ & $1,294,966$ & $1,579,426$ & 81.99 \\
\hline OKLAHOMA & + & 15,478 & $1,654,370$ & $1,669,848$ & $2,019,939$ & 82.67 \\
\hline OKLAHOMA STATE & + & 22,750 & 169,083 & 191,833 & 398,341 & 48.16 \\
\hline PENNSYLVANIA & G & 0 & $2,328,841$ & $2,328,841$ & $2,522,755$ & 92.31 \\
\hline PENNSYLVANIA STATE & bG+ & 0 & $1,367,657$ & $1,367,657$ & $1,396,990$ & 97.90 \\
\hline QUEEN'S & G+ & . & $1,484,944$ & $1,484,944$ & $1,658,452$ & 89.54 \\
\hline ROCHESTER & $b+$ & 36,879 & $1,808,943$ & $1,845,822$ & $1,992,560$ & 92.64 \\
\hline SASKATCHEWAN & G+ & . & . & . & $2,488,014$ & . \\
\hline SOUTH CAROLINA & G & 18,000 & 630,840 & 648,840 & 676,024 & 95.98 \\
\hline SOUTHERN CALIFORNIA & + & 247,453 & $2,309,928$ & $2,557,381$ & $3,063,445$ & 83.48 \\
\hline SOUTHERN ILLINOIS & G & 0 & 143,343 & 143,343 & 759,892 & 18.86 \\
\hline SUNY-STONY BROOK & bG & 5,484 & $1,456,954$ & $1,462,438$ & $1,552,969$ & 94.17 \\
\hline TEMPLE & $b+$ & 71,927 & $2,037,357$ & $2,109,284$ & $2,420,209$ & 87.15 \\
\hline TENNESSEE & $b+$ & 3,300 & $1,414,771$ & $1,418,071$ & $1,609,771$ & 88.09 \\
\hline TEXAS TECH & bG+ & 259,419 & $2,032,908$ & $2,292,327$ & $3,016,810$ & 75.99 \\
\hline TULANE & G+ & 7,062 & $1,431,303$ & $1,438,365$ & $1,438,365$ & 100.00 \\
\hline UTAH & $\mathrm{b}$ & 25,050 & 839,926 & 864,976 & $1,145,847$ & 75.49 \\
\hline VANDERBILT & + & 277,574 & $2,409,820$ & $2,687,394$ & $2,721,911$ & 98.73 \\
\hline VIRGINIA & G+ & 0 & $1,438,726$ & $1,438,726$ & $1,889,113$ & 76.16 \\
\hline WASHINGTON & + & 7,894 & $2,616,675$ & $2,624,569$ & $2,766,258$ & 94.88 \\
\hline WASHINGTON U.-ST. LOUIS & $b+$ & 114,246 & $3,072,929$ & $3,187,175$ & $3,460,245$ & 92.11 \\
\hline WISCONSIN & $\mathrm{b}$ & 0 & $1,433,304$ & $1,433,304$ & $1,452,998$ & 98.64 \\
\hline
\end{tabular}




\section{Electronic Resources Expenditures}

\begin{tabular}{|c|c|c|c|c|c|}
\hline Notes & $\begin{array}{r}\text { One-time } \\
\text { Electronic } \\
\text { Resource } \\
\text { Purchases } \\
22\end{array}$ & $\begin{array}{r}\text { Ongoing } \\
\text { Electronic } \\
\text { Resource } \\
\text { Purchases } \\
23\end{array}$ & $\begin{array}{r}\text { Total Electronic } \\
\text { Resource } \\
\text { Purchases } \\
22+23\end{array}$ & $\begin{array}{r}\text { Total Library } \\
\text { Materials } \\
\text { Expenditures } \\
16\end{array}$ & $\begin{array}{r}\text { Electronic } \\
\text { Resources as a } \\
\% \text { of Library } \\
\text { Materials } \\
\text { Budget }\end{array}$ \\
\hline+ & 108,713 & $2,116,106$ & $2,224,819$ & $2,400,807$ & 92.67 \\
\hline
\end{tabular}

+ - See Footnotes

- Unavailable, not applicable or no data supplied
G - Government documents not included in serials counts b - Basis of volume count is bibliographic

\section{Summary Data}

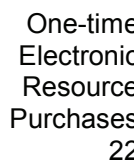

Ongoing

Electronic

Resource

Purchases

23
Total Electronic

Resource

Purchases

$22+23$
Total Library

Materials

Expenditures

16
Electronic Resources as a \% of Library Materials

Budget

\begin{tabular}{lrrrrr}
\hline Mean & 67,196 & $1,526,414$ & $1,575,073$ & $1,879,927$ & 84.15 \\
Median & 37,178 & $1,447,840$ & $1,459,592$ & 88.79 \\
High & 277,574 & $3,563,846$ & $3,692,727$ & $4,223,445$ \\
Low & 667 & 83,285 & 83,285 & 131,954 \\
Totals & $2,822,248$ & $88,531,989$ & $91,354,237$ & $114,675,574$ \\
Number of Libraries Reporting & 42 & 58 & 58 & 61
\end{tabular}




\section{Electronic Resources Expenditures}

\begin{tabular}{|c|c|c|c|c|c|}
\hline & Notes & $\begin{array}{r}\text { Bibl. Utilities, } \\
\text { Networks, etc. } \\
\text { Library } \\
\text { Expenditures } \\
24 a\end{array}$ & $\begin{array}{r}\text { Bibl. Utilities, } \\
\text { Networks, etc. } \\
\text { External } \\
\text { Expenditures } \\
24 \mathrm{~b}\end{array}$ & $\begin{array}{r}\text { Computer } \\
\text { Hardware and } \\
\text { Software } \\
\text { Expenditures } \\
25\end{array}$ & $\begin{array}{r}\text { Document Delivery } \\
\text { / Interlibrary Loan } \\
\text { Expenditures } \\
26\end{array}$ \\
\hline ALABAMA & $b+$ & 799 & 0 & 1,546 & 1,340 \\
\hline ARIZONA & $b+$ & 0 & 957,021 & 19,692 & 2,814 \\
\hline BOSTON & bG & 5,180 & & 68,149 & 19,232 \\
\hline CALIFORNIA, DAVIS & + & 11,350 & 0 & . & 59,298 \\
\hline CALIFORNIA, IRVINE & $b+$ & 14,249 & 11,750 & 14,021 & 22,822 \\
\hline CALIFORNIA, LOS ANGELES & $b+$ & . & . & . & \\
\hline CALIFORNIA, SAN DIEGO & + & 0 & 254,003 & 166,053 & 3,189 \\
\hline CASE WESTERN RESERVE & + & 4,427 & 0 & 36,313 & 8,260 \\
\hline CHICAGO & + & . & . & 125,000 & 21,222 \\
\hline CINCINNATI & $b+$ & 10,000 & 0 & 158,562 & 202 \\
\hline COLUMBIA & $\mathrm{G}+$ & 5,547 & . & 45,430 & 0 \\
\hline CONNECTICUT & $\mathrm{b}$ & 13,077 & 0 & 68,077 & 3,690 \\
\hline CORNELL & $\mathrm{G}+$ & 11,810 & 0 & 111,081 & 6,581 \\
\hline DARTMOUTH & $b+$ & . & . & 15,679 & 83,511 \\
\hline DUKE & bG & 82,016 & 0 & 90,551 & 260 \\
\hline EMORY & $\mathrm{G}+$ & 4,005 & 0 & 54,775 & 6,676 \\
\hline FLORIDA & $\mathrm{G}+$ & 2,950 & . & 79,631 & 4,825 \\
\hline FLORIDA STATE & bG+ & 0 & 0 & 5,756 & 1,146 \\
\hline GEORGE WASHINGTON & bG+ & 4,662 & 0 & 93,536 & 6,709 \\
\hline GEORGETOWN & bG+ & 3,250 & 0 & 62,578 & 3,584 \\
\hline HARVARD & $\mathrm{b}$ & 920 & 0 & 147,886 & 13,581 \\
\hline HAWAII & & 524 & 0 & 21,891 & \\
\hline HOWARD & $\mathrm{b}$ & 0 & 4,500 & 30,000 & 8,500 \\
\hline ILLINOIS, CHICAGO & + & . & . & 33,274 & 34,692 \\
\hline INDIANA & $\mathrm{b}$ & 0 & 0 & 0 & 0 \\
\hline IOWA & $\mathrm{G}+$ & 15,309 & 0 & 2,664 & 48,844 \\
\hline KANSAS & $\mathrm{G}+$ & 0 & 0 & 71,695 & 21,210 \\
\hline KENTUCKY & + & 10,800 & 0 & 26,096 & 36,442 \\
\hline LOUISIANA STATE & $\mathrm{G}+$ & 0 & 0 & 0 & 2,514 \\
\hline MCMASTER & + & 23,683 & 482 & 33,598 & 24,100 \\
\hline
\end{tabular}




\section{Electronic Resources Expenditures}

\begin{tabular}{|c|c|c|c|c|c|}
\hline & Notes & $\begin{array}{r}\text { Bibl. Utilities, } \\
\text { Networks, etc. } \\
\text { Library } \\
\text { Expenditures } \\
24 a\end{array}$ & $\begin{array}{r}\text { Bibl. Utilities, } \\
\text { Networks, etc. } \\
\text { External } \\
\text { Expenditures } \\
24 \mathrm{~b}\end{array}$ & $\begin{array}{r}\text { Computer } \\
\text { Hardware and } \\
\text { Software } \\
\text { Expenditures } \\
25\end{array}$ & $\begin{array}{r}\text { Document Delivery } \\
\text { / Interlibrary Loan } \\
\text { Expenditures } \\
26\end{array}$ \\
\hline MIAMI & + & 8,068 & 0 & 29,937 & 21,136 \\
\hline MICHIGAN & $b$ & 0 & 124,000 & 0 & 0 \\
\hline MINNESOTA & $b+$ & . & . & 23,188 & 30,996 \\
\hline MONTREAL & + & 0 & 0 & 0 & 0 \\
\hline NEW MEXICO & + & 262,022 & 0 & 73,643 & 10,854 \\
\hline NEW YORK & $b+$ & 16,672 & . & 64,123 & 114,967 \\
\hline NORTH CAROLINA & $\mathrm{G}+$ & . & . & . & . \\
\hline NORTHWESTERN & + & 6,583 & . & 14,707 & 2,622 \\
\hline OHIO STATE & $b+$ & 16,030 & . & 164,319 & 11,698 \\
\hline OKLAHOMA & + & 23,567 & 34,987 & 34,171 & 44,710 \\
\hline OKLAHOMA STATE & + & 5,090 & 1,754 & 13,093 & 771 \\
\hline PENNSYLVANIA & G & 0 & 0 & 15,822 & 90,339 \\
\hline PENNSYLVANIA STATE & bG+ & 6,884 & 0 & 31,948 & \\
\hline QUEEN'S & G+ & . & . & . & . \\
\hline ROCHESTER & $b+$ & 1,611 & 0 & 167,468 & 14,070 \\
\hline SASKATCHEWAN & G+ & . & . & . & . \\
\hline SOUTH CAROLINA & G & 1,836 & 0 & 60,947 & 3,077 \\
\hline SOUTHERN CALIFORNIA & + & 8,697 & 0 & 235,812 & 9,262 \\
\hline SOUTHERN ILLINOIS & G & 0 & 0 & 10,523 & 4,686 \\
\hline SUNY-STONY BROOK & bG & 18,046 & 349,837 & 285,213 & 8,085 \\
\hline TEMPLE & $b+$ & 3,718 & 0 & 85,823 & 3,630 \\
\hline TENNESSEE & $b+$ & 6,266 & 0 & 21,286 & 572 \\
\hline TEXAS TECH & bG+ & 48,398 & 0 & 55,479 & 11,043 \\
\hline TULANE & G+ & 2,629 & 0 & 36,519 & 7,518 \\
\hline UTAH & $\mathrm{b}$ & 0 & 29,643 & 58,983 & 3,549 \\
\hline VANDERBILT & + & 4,134 & . & 86,724 & 9,494 \\
\hline VIRGINIA & G+ & 3,217 & 0 & 104,653 & 20,791 \\
\hline WASHINGTON & + & 0 & 0 & 37,709 & 22,504 \\
\hline WASHINGTON U.-ST. LOUIS & $b+$ & . & 355 & 150,281 & 1,975 \\
\hline WISCONSIN & $\mathrm{b}$ & . & . & 20,807 & 205,637 \\
\hline
\end{tabular}

30 - ARL Academic Health Sciences Library Statistics 2009-2010 


\section{Electronic Resources Expenditures}

\begin{tabular}{|c|c|c|c|c|c|}
\hline & Notes & $\begin{array}{r}\text { Bibl. Utilities, } \\
\text { Networks, etc. } \\
\text { Library } \\
\text { Expenditures } \\
24 a\end{array}$ & $\begin{array}{r}\text { Bibl. Utilities, } \\
\text { Networks, etc. } \\
\text { External } \\
\text { Expenditures } \\
24 \mathrm{~b}\end{array}$ & $\begin{array}{r}\text { Computer } \\
\text { Hardware and } \\
\text { Software } \\
\text { Expenditures } \\
25\end{array}$ & $\begin{array}{r}\text { Document Delivery } \\
\text { / Interlibrary Loan } \\
\text { Expenditures } \\
26\end{array}$ \\
\hline YALE & + & 5,280 & . & 45,564 & 9,686 \\
\hline
\end{tabular}

\section{Summary Data}

Bibl. Utilities, Networks, etc. Library Expenditures

$24 a$
Bibl. Utilities, Networks, etc. External Expenditures
Computer Hardware and

Software Expenditures

25
Document Delivery / Interlibrary Loan Expenditures

\begin{tabular}{lrrr}
\hline Mean & 17,719 & 160,757 & 67,544 \\
Median & 6,425 & 29,643 & 50,170 \\
High & 262,022 & 957,021 & 28,262 \\
Low & 524,213 & 205,637 \\
Totals & 673,306 & 355 & 1,546 \\
Number of Libraries Reporting & 38 & $1,768,332$ & $3,512,276$ \\
\end{tabular}


Personnel and Public Services

$\begin{array}{rrrrrrr}\text { Notes } & \text { Professional } & \text { Support Staff } & \text { Student } & \text { Total Staff } & \text { Staffed } & \text { Library } \\ & \text { Staff (FTE) } & \text { (FTE) } & \text { Assistants } & \text { (FTE) } & \text { Service } & \text { Service } \\ 27 \mathrm{a} & 27 \mathrm{~b} & (\text { FTE) } & 27 & \text { Points } & \text { Hours } \\ & & 27 \mathrm{c} & & 28 & 29\end{array}$

\begin{tabular}{|c|c|c|c|c|c|c|c|}
\hline ALABAMA & $b+$ & 2 & 3 & 1 & 6 & 2 & 63 \\
\hline ARIZONA & $b+$ & 15 & 13 & 5 & 33 & 1 & 109 \\
\hline CALIFORNIA, DAVIS & + & 7 & 14 & 4 & 25 & 2 & 86 \\
\hline CALIFORNIA, LOS ANGELES & $b+$ & 10 & 11 & 11 & 32 & 1 & 84 \\
\hline CALIFORNIA, SAN DIEGO & + & 10 & 16 & 5 & 31 & 3 & 90 \\
\hline CASE WESTERN RESERVE & + & 7 & 10 & 9 & 26 & 2 & . \\
\hline CONNECTICUT & $\mathrm{b}$ & 15 & 13 & 3 & 31 & 3 & 94 \\
\hline CORNELL & G+ & 11 & 16 & 0 & 27 & 2 & 108 \\
\hline DARTMOUTH & $b+$ & 7 & 12 & 3 & 22 & 4 & 110 \\
\hline DUKE & bG & 23 & 7 & 0 & 30 & 3 & 68 \\
\hline EMORY & G+ & 11 & 15 & 0 & 26 & 6 & 91 \\
\hline FLORIDA & G+ & 19 & 27 & 11 & 57 & 2 & 97 \\
\hline HAWAII & & 5 & 3 & . & 8 & 1 & 78 \\
\hline HOWARD & $\mathrm{b}$ & 4 & 13 & 5 & 22 & 3 & 113 \\
\hline ILLINOIS, CHICAGO & + & 22 & 28 & 4 & 54 & 5 & 102 \\
\hline INDIANA & $\mathrm{b}$ & 9 & 18 & 3 & 30 & 3 & 40 \\
\hline IOWA & G+ & 15 & 14 & 9 & 38 & 3 & 99 \\
\hline KANSAS & G+ & 12 & 19 & 3 & 34 & 1 & 87 \\
\hline KENTUCKY & + & 13 & 6 & 8 & 27 & 3 & 110 \\
\hline LOUISIANA STATE & G+ & 1 & 3 & 2 & 6 & 1 & 88 \\
\hline MCMASTER & + & 8 & 17 & 4 & 29 & 3 & 97 \\
\hline
\end{tabular}




\section{Personnel and Public Services}

$\begin{array}{rrrrrrr}\text { Notes } & \text { Professional } & \text { Support Staff } & \text { Student } & \text { Total Staff } & \text { Staffed } & \text { Library } \\ & \text { Staff (FTE) } & (\text { FTE) } & \text { Assistants } & \text { (FTE) } & \text { Service } & \text { Service } \\ 27 a & 27 b & (\text { FTE) } & 27 & \text { Points } & \text { Hours } \\ & & 27 \mathrm{c} & & 28 & 29\end{array}$

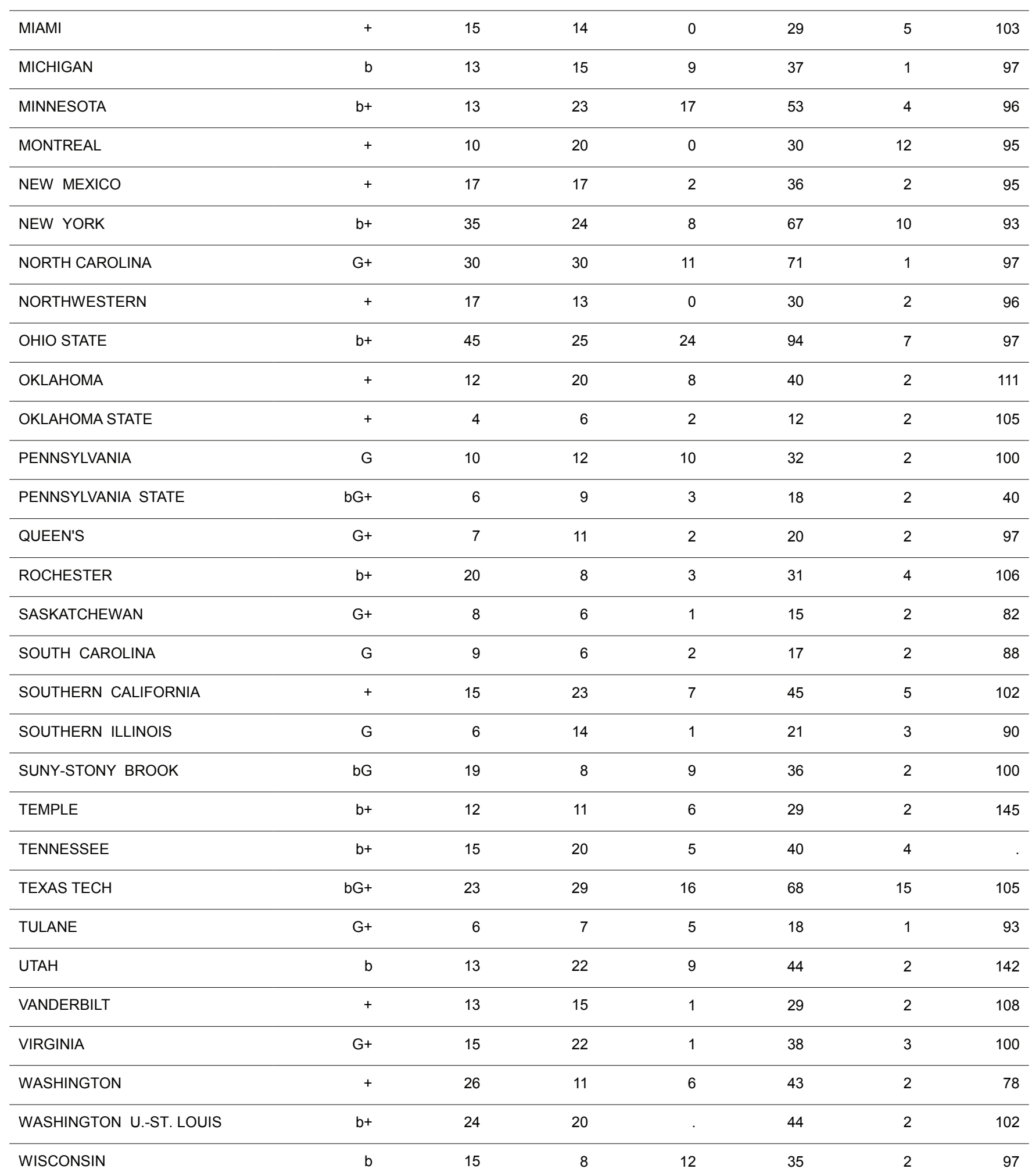




\section{Personnel and Public Services}

\begin{tabular}{|c|c|c|c|c|c|c|c|}
\hline & Notes & $\begin{array}{r}\text { Professional } \\
\text { Staff (FTE) } \\
27 a\end{array}$ & $\begin{array}{r}\text { Support Staff } \\
\text { (FTE) } \\
27 \mathrm{~b}\end{array}$ & $\begin{array}{r}\text { Student } \\
\text { Assistants } \\
(\mathrm{FTE}) \\
27 \mathrm{c}\end{array}$ & $\begin{array}{r}\text { Total Staff } \\
(\text { FTE) } \\
27\end{array}$ & $\begin{array}{r}\text { Staffed } \\
\text { Service } \\
\text { Points } \\
28\end{array}$ & $\begin{array}{r}\text { Library } \\
\text { Service } \\
\text { Hours } \\
29\end{array}$ \\
\hline YALE & + & 19 & 16 & 1 & 36 & 2 & 105 \\
\hline - Unavailable, not applicable or no data supplied & b-Basis & volume count is & ibliographic & & & & \\
\hline
\end{tabular}

\section{Summary Data}

$\begin{array}{rrrrrr}\text { Professional } & \text { Support Staff } & \text { Student } & \text { Total Staff (FTE) } & \text { Staffed Service } & \text { Library Service } \\ \text { Staff (FTE) } & (\text { FTE) } & \text { Assistants (FTE) } & 27 & \text { Points } & \text { Hours } \\ 27 a & 27 b & 27 c & & 28 & 29\end{array}$

\begin{tabular}{|c|c|c|c|c|c|c|}
\hline Mean & 14 & 14 & 6 & 34 & 3 & 97 \\
\hline Median & 13 & 13 & 5 & 30 & 2 & 97 \\
\hline High & 76 & 40 & 24 & 121 & 15 & 168 \\
\hline Low & 1 & 3 & 1 & 6 & 1 & 40 \\
\hline Totals & 875 & 876 & 310 & 2,061 & 188 & 5,752 \\
\hline Number of Libraries & 61 & 61 & 52 & 61 & 60 & 59 \\
\hline
\end{tabular}


Personnel and Public Services

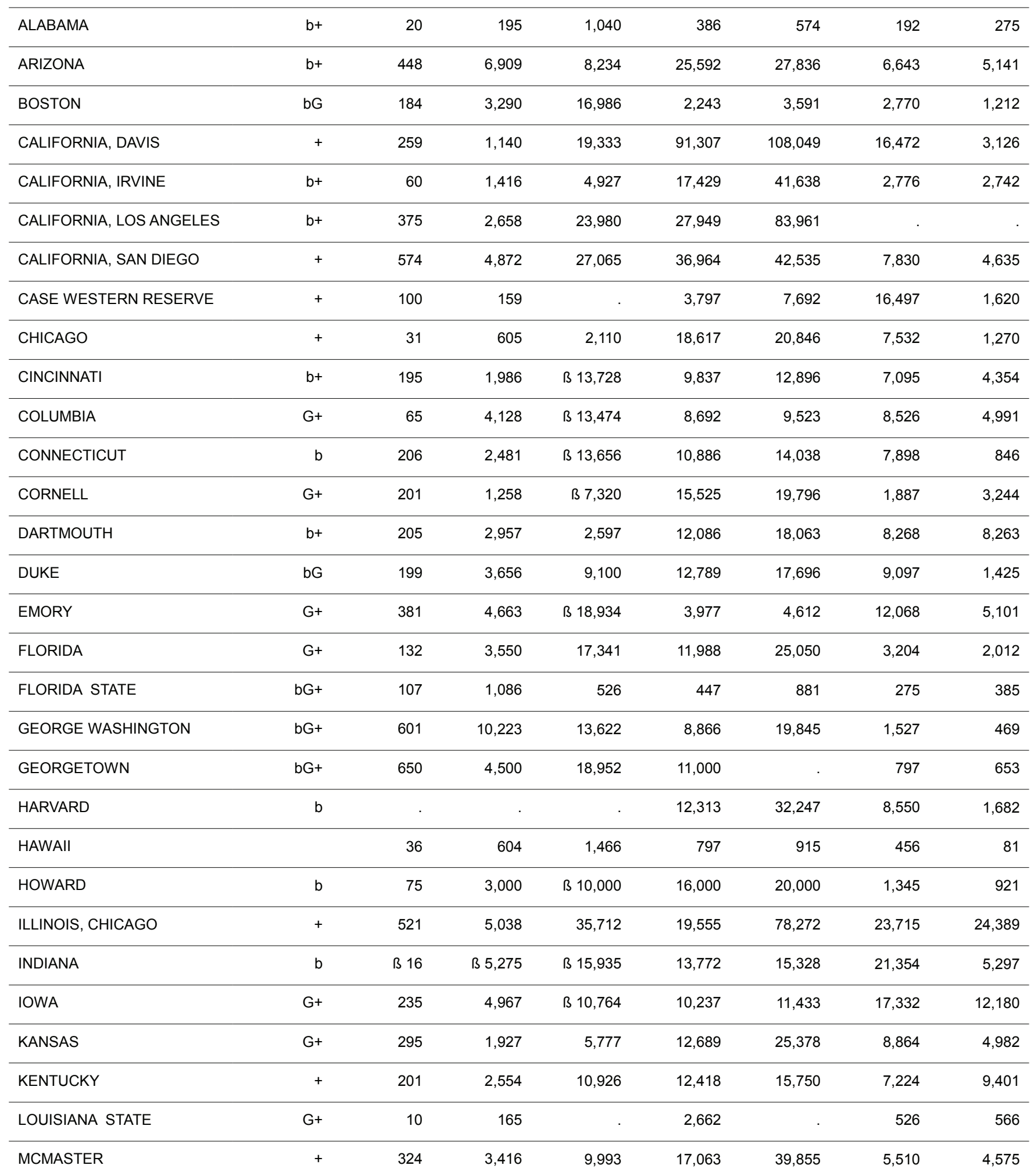


Personnel and Public Services

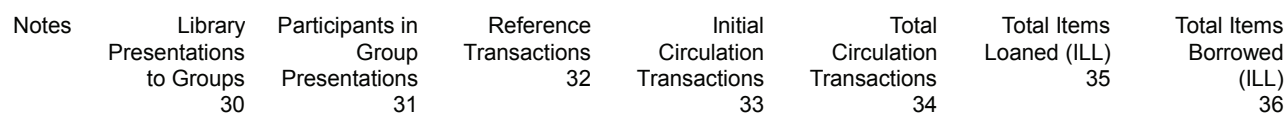

\begin{tabular}{|c|c|c|c|c|c|c|c|c|}
\hline MIAMI & + & 255 & 2,760 & 9,485 & 4,660 & 6,527 & 13,556 & 1,551 \\
\hline MICHIGAN & $b$ & 151 & 2,531 & 14,709 & 20,137 & 35,862 & 0 & 0 \\
\hline MINNESOTA & $b+$ & 264 & 5,309 & 16,485 & 21,875 & 34,383 & 48,643 & 7,205 \\
\hline MONTREAL & + & 303 & 4,276 & 8,800 & 16,389 & 35,122 & 6,763 & 1,720 \\
\hline NEW MEXICO & + & 697 & 6,025 & ß5,638 & 8,724 & 15,399 & 1,321 & 1,803 \\
\hline NEW YORK & $b+$ & 178 & 3,465 & ß 842 & 6,615 & 7,265 & 7,003 & 8,390 \\
\hline NORTH CAROLINA & G+ & 315 & 6,374 & 17,305 & 69,754 & 82,489 & 28,255 & 2,848 \\
\hline NORTHWESTERN & + & 246 & 3,170 & 4,185 & 10,269 & 21,250 & 11,093 & 6,089 \\
\hline OHIO STATE & $b+$ & 148 & 1,977 & 7,488 & 10,731 & 30,100 & 14,304 & 4,078 \\
\hline OKLAHOMA & + & 61 & 1,105 & 11,369 & 4,798 & 4,798 & 7,310 & 3,204 \\
\hline OKLAHOMA STATE & + & 31 & 368 & 967 & 4,921 & 5,520 & 2,170 & 2,258 \\
\hline PENNSYLVANIA & G & 140 & 1,879 & . & 6,836 & 18,172 & 8,877 & 23,557 \\
\hline PENNSYLVANIA STATE & bG+ & 68 & 1,513 & 6,917 & 8,539 & 10,505 & 7,254 & 1,360 \\
\hline QUEEN'S & G+ & 289 & 5,084 & 6,372 & 7,749 & 8,662 & 4,305 & 1,411 \\
\hline ROCHESTER & $b+$ & 191 & 1,818 & ß 9,220 & 4,095 & 5,474 & 3,951 & 4,485 \\
\hline SASKATCHEWAN & G+ & 199 & 1,313 & 2,682 & 14,516 & 22,392 & 2,776 & 1,969 \\
\hline SOUTH CAROLINA & G & 50 & 1,126 & 1,031 & 1,179 & 1,931 & 1,332 & 1,253 \\
\hline SOUTHERN CALIFORNIA & + & 239 & 3,617 & 8,461 & 18,241 & 23,797 & 10,937 & 1,098 \\
\hline SOUTHERN ILLINOIS & G & 134 & 767 & 3,888 & 25,436 & 29,925 & 6,810 & 2,409 \\
\hline SUNY-STONY BROOK & bG & 196 & 2,463 & 10,364 & 3,990 & 5,934 & 4,728 & 765 \\
\hline TEMPLE & $b+$ & 63 & 949 & 3,655 & 4,941 & 6,809 & 1,264 & 1,087 \\
\hline TENNESSEE & $b+$ & 334 & 3,776 & ß 17,466 & 3,155 & 4,696 & 3,213 & 1,734 \\
\hline TEXAS TECH & bG+ & 462 & 4,226 & 16,311 & 29,142 & 74,179 & 6,429 & 3,088 \\
\hline TULANE & $\mathrm{G}+$ & 84 & 464 & 6,224 & 7,651 & 9,451 & 3,230 & 3,426 \\
\hline UTAH & $b$ & 201 & 2,436 & B 32,000 & 7,153 & 7,690 & 3,325 & 6,113 \\
\hline VANDERBILT & + & 375 & 11,721 & 3,756 & 9,828 & 10,604 & 7,396 & 3,083 \\
\hline VIRGINIA & G+ & 482 & 3,578 & ß 9,364 & 7,514 & 9,882 & 4,633 & 2,514 \\
\hline WASHINGTON & + & 156 & 3,081 & ß 4,744 & 16,004 & 55,540 & 8,548 & 7,268 \\
\hline WASHINGTON U.-ST. LOUIS & $b+$ & В 143 & В 1,729 & ß 9,145 & 21,025 & 25,860 & 21,670 & 2,142 \\
\hline WISCONSIN & $b$ & 230 & 3,547 & 2,461 & 12,752 & 17,074 & 6,031 & 22,985 \\
\hline
\end{tabular}

36 - ARL Academic Health Sciences Library Statistics 2009-2010 


\section{Personnel and Public Services}

\begin{tabular}{|c|c|c|c|c|c|c|c|}
\hline Notes & $\begin{array}{r}\text { Library } \\
\text { Presentations } \\
\text { to Groups } \\
30\end{array}$ & $\begin{array}{r}\text { Participants in } \\
\text { Group } \\
\text { Presentations } \\
31\end{array}$ & $\begin{array}{r}\text { Reference } \\
\text { Transactions } \\
32\end{array}$ & $\begin{array}{r}\text { Initial } \\
\text { Circulation } \\
\text { Transactions } \\
33\end{array}$ & $\begin{array}{r}\text { Total } \\
\text { Circulation } \\
\text { Transactions } \\
34\end{array}$ & $\begin{array}{r}\text { Total Items } \\
\text { Loaned (ILL) } \\
35\end{array}$ & $\begin{array}{r}\text { Total Items } \\
\text { Borrowed } \\
\text { (ILL) } \\
36\end{array}$ \\
\hline
\end{tabular}

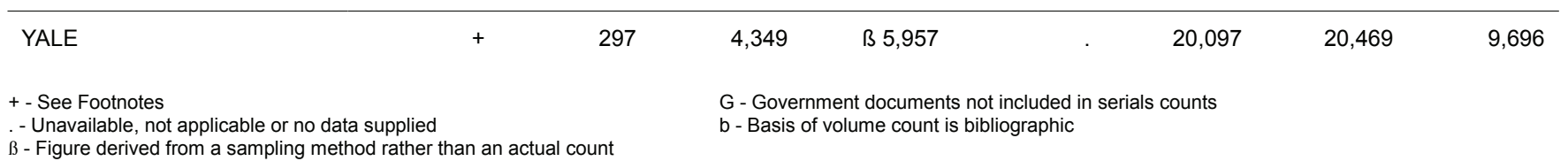

\section{Summary Data}

\begin{tabular}{|c|c|c|c|c|c|c|}
\hline $\begin{array}{r}\text { Library } \\
\text { Presentations } \\
\text { to Groups } \\
30\end{array}$ & $\begin{array}{r}\text { Participants } \\
\text { in Group } \\
\text { Presentations } \\
31\end{array}$ & $\begin{array}{r}\text { Reference } \\
\text { Transactions } \\
32\end{array}$ & $\begin{array}{r}\text { Initial } \\
\text { Circulation } \\
\text { Transactions } \\
33\end{array}$ & $\begin{array}{r}\text { Total } \\
\text { Circulation } \\
\text { Transactions } \\
34\end{array}$ & $\begin{array}{r}\text { Total Items } \\
\text { Loaned (ILL) } \\
35\end{array}$ & $\begin{array}{r}\text { Total Items } \\
\text { Borrowed } \\
\text { (ILL) } \\
36\end{array}$ \\
\hline 228 & 3,091 & 10,365 & 13,942 & 23,147 & 8,370 & 4,346 \\
\hline 200 & 2,859 & 9,145 & 10,809 & 17,696 & 7,003 & 2,742 \\
\hline 697 & 11,721 & 35,712 & 91,307 & 108,049 & 48,643 & 24,389 \\
\hline 10 & 159 & 526 & 386 & 574 & 192 & 81 \\
\hline 13,688 & 185,474 & 590,789 & 836,502 & $1,365,689$ & 493,826 & 256,427 \\
\hline 60 & 60 & 57 & 60 & 59 & 59 & 59 \\
\hline
\end{tabular}




\section{Rank Order Table 1: Volumes in Library}

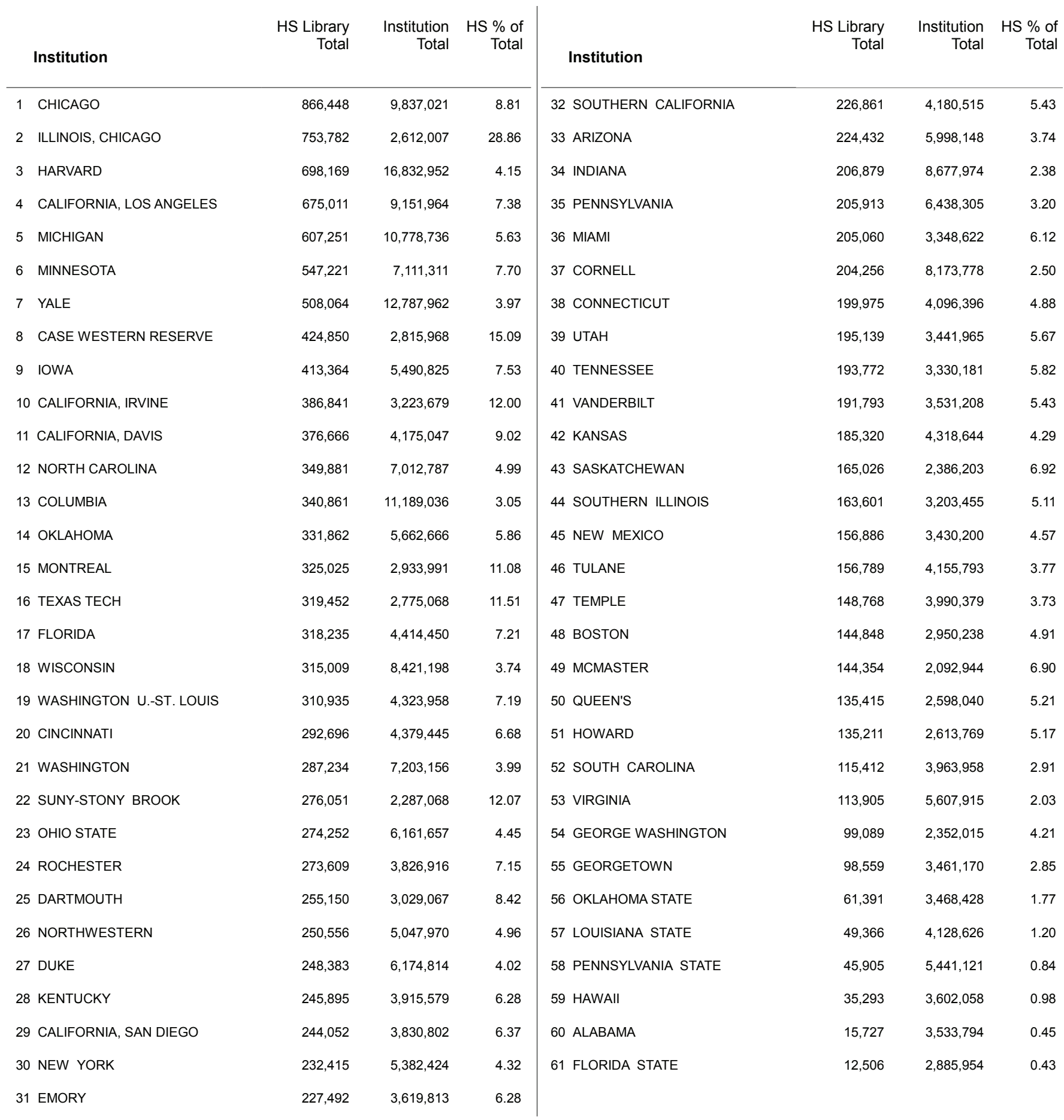


Rank Order Table 2: Volumes Added (Gross)

\begin{tabular}{|c|c|c|c|c|c|c|c|c|}
\hline & Institution & $\begin{array}{r}\text { HS Library } \\
\text { Total }\end{array}$ & $\begin{array}{r}\text { Institution } \\
\text { Total }\end{array}$ & $\begin{array}{r}\text { HS \% of } \\
\text { Total }\end{array}$ & Institution & $\begin{array}{r}\text { HS Library } \\
\text { Total }\end{array}$ & $\begin{array}{r}\text { Institution } \\
\text { Total }\end{array}$ & $\begin{array}{r}\text { HS \% of } \\
\text { Total }\end{array}$ \\
\hline 1 & IOWA & 61,962 & 232,867 & 26.61 & 32 VANDERBILT & 1,907 & 80,884 & 2.36 \\
\hline 2 & MICHIGAN & 44,915 & 250,652 & 17.92 & 33 SUNY-STONY BROOK & 1,717 & 13,097 & 13.11 \\
\hline 3 & YALE & 19,766 & 237,514 & 8.32 & 34 OHIO STATE & 1,676 & 197,966 & 0.85 \\
\hline 4 & ILLINOIS, CHICAGO & 19,507 & 204,412 & 9.54 & 35 HAWAll & 1,650 & 40,170 & 4.11 \\
\hline 5 & CHICAGO & 16,890 & 529,024 & 3.19 & 36 WISCONSIN & 1,575 & 124,316 & 1.27 \\
\hline 6 & WASHINGTON U.-ST. LOUIS & 16,133 & 93,598 & 17.24 & 37 DUKE & 1,525 & 93,272 & 1.64 \\
\hline 7 & CALIFORNIA, IRVINE & 14,185 & 118,218 & 12.00 & 38 PENNSYLVANIA & 1,524 & 222,613 & 0.68 \\
\hline 8 & KANSAS & 10,918 & 54,858 & 19.90 & 39 TENNESSEE & 1,518 & 47,669 & 3.18 \\
\hline 9 & NEW YORK & 6,477 & 97,075 & 6.67 & 40 QUEEN'S & 1,364 & 32,676 & 4.17 \\
\hline 10 & MONTREAL & 6,312 & 55,224 & 11.43 & 41 BOSTON & 1,314 & 40,862 & 3.22 \\
\hline 11 & CALIFORNIA, LOS ANGELES & 5,776 & 188,975 & 3.06 & 42 GEORGE WASHINGTON & 1,218 & 96,790 & 1.26 \\
\hline 12 & TEXAS TECH & 5,464 & 49,550 & 11.03 & 43 CASE WESTERN RESERVE & 1,143 & 44,271 & 2.58 \\
\hline 13 & CALIFORNIA, SAN DIEGO & 5,136 & 208,536 & 2.46 & 44 CINCINNATI & 1,126 & 110,457 & 1.02 \\
\hline 14 & MINNESOTA & 5,002 & 131,822 & 3.79 & 45 VIRGINIA & 1,070 & 112,250 & 0.95 \\
\hline 15 & KENTUCKY & 4,322 & 85,179 & 5.07 & 46 OKLAHOMA STATE & 999 & 55,393 & 1.80 \\
\hline 16 & TEMPLE & 3,934 & 57,417 & 6.85 & 47 CORNELL & 988 & 117,628 & 0.84 \\
\hline 17 & CALIFORNIA, DAVIS & 3,714 & 57,292 & 6.48 & 48 DARTMOUTH & 964 & 193,275 & 0.50 \\
\hline 18 & SOUTHERN CALIFORNIA & 3,192 & 82,671 & 3.86 & 49 NEW MEXICO & 913 & 50,532 & 1.81 \\
\hline 19 & NORTH CAROLINA & 3,167 & 207,232 & 1.53 & 50 ARIZONA & 680 & 104,581 & 0.65 \\
\hline 20 & OKLAHOMA & 2,806 & 121,400 & 2.31 & 51 TULANE & 618 & 159,849 & 0.39 \\
\hline 21 & MCMASTER & 2,790 & 115,033 & 2.43 & 52 SOUTH CAROLINA & 574 & 32,894 & 1.75 \\
\hline 22 & HARVARD & 2,754 & 275,950 & 1.00 & 53 MIAMI & 532 & 87,602 & 0.61 \\
\hline 23 & ROCHESTER & 2,713 & 29,257 & 9.27 & 54 FLORIDA & 500 & 122,873 & 0.41 \\
\hline 24 & CONNECTICUT & 2,631 & 143,221 & 1.84 & 55 UTAH & 459 & 27,164 & 1.69 \\
\hline 25 & INDIANA & 2,527 & 152,934 & 1.65 & 56 HOWARD & 368 & 15,826 & 2.33 \\
\hline 26 & COLUMBIA & 2,425 & 535,687 & 0.45 & 57 ALABAMA & 333 & 158,565 & 0.21 \\
\hline 27 & EMORY & 2,319 & 82,601 & 2.81 & 58 PENNSYLVANIA STATE & 331 & 197,064 & 0.17 \\
\hline 28 & WASHINGTON & 2,030 & 208,765 & 0.97 & 59 LOUISIANA STATE & 277 & 19,872 & 1.39 \\
\hline 29 & SOUTHERN ILLINOIS & 1,952 & 43,163 & 4.52 & 60 GEORGETOWN & 217 & 67,215 & 0.32 \\
\hline 30 & NORTHWESTERN & 1,931 & 169,105 & 1.14 & 61 FLORIDA STATE & 216 & 42,233 & 0.51 \\
\hline 31 & SASKATCHEWAN & 1,926 & 28,103 & 6.85 & & & & \\
\hline
\end{tabular}


Rank Order Table 3: Current Serials (Total)

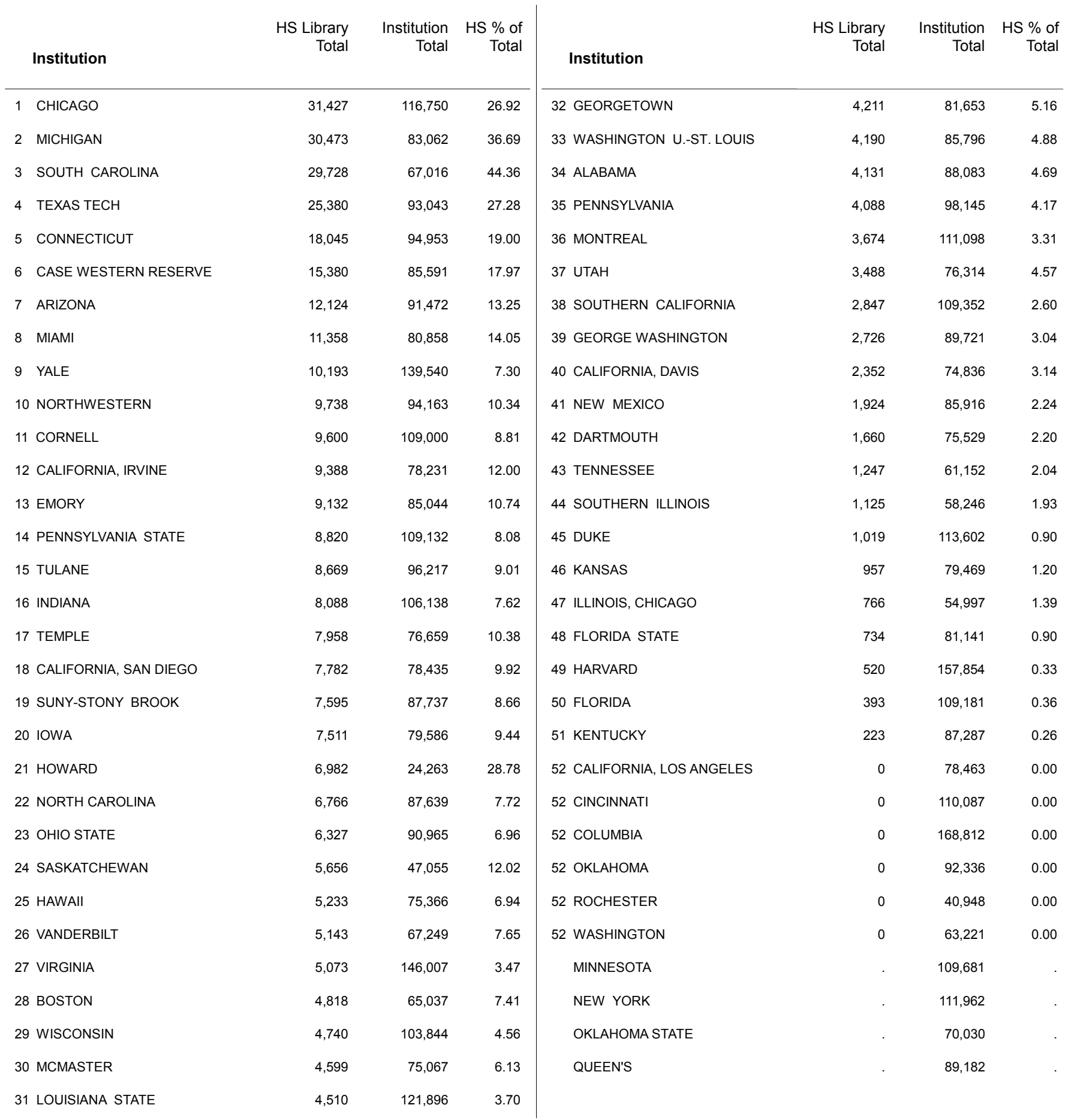


Rank Order Table 4: Total Library Expenditures

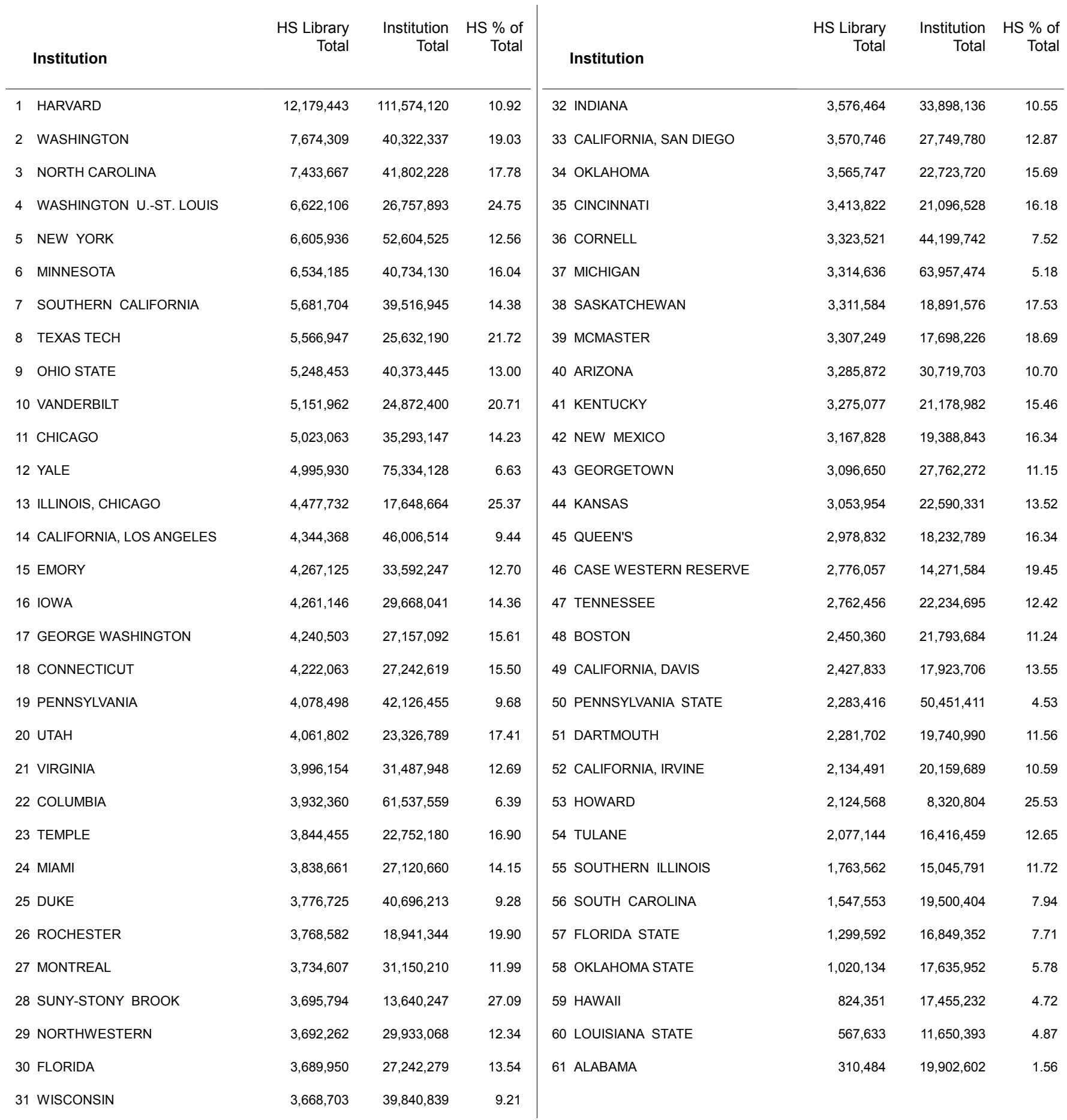


Rank Order Table 5: Total Staff

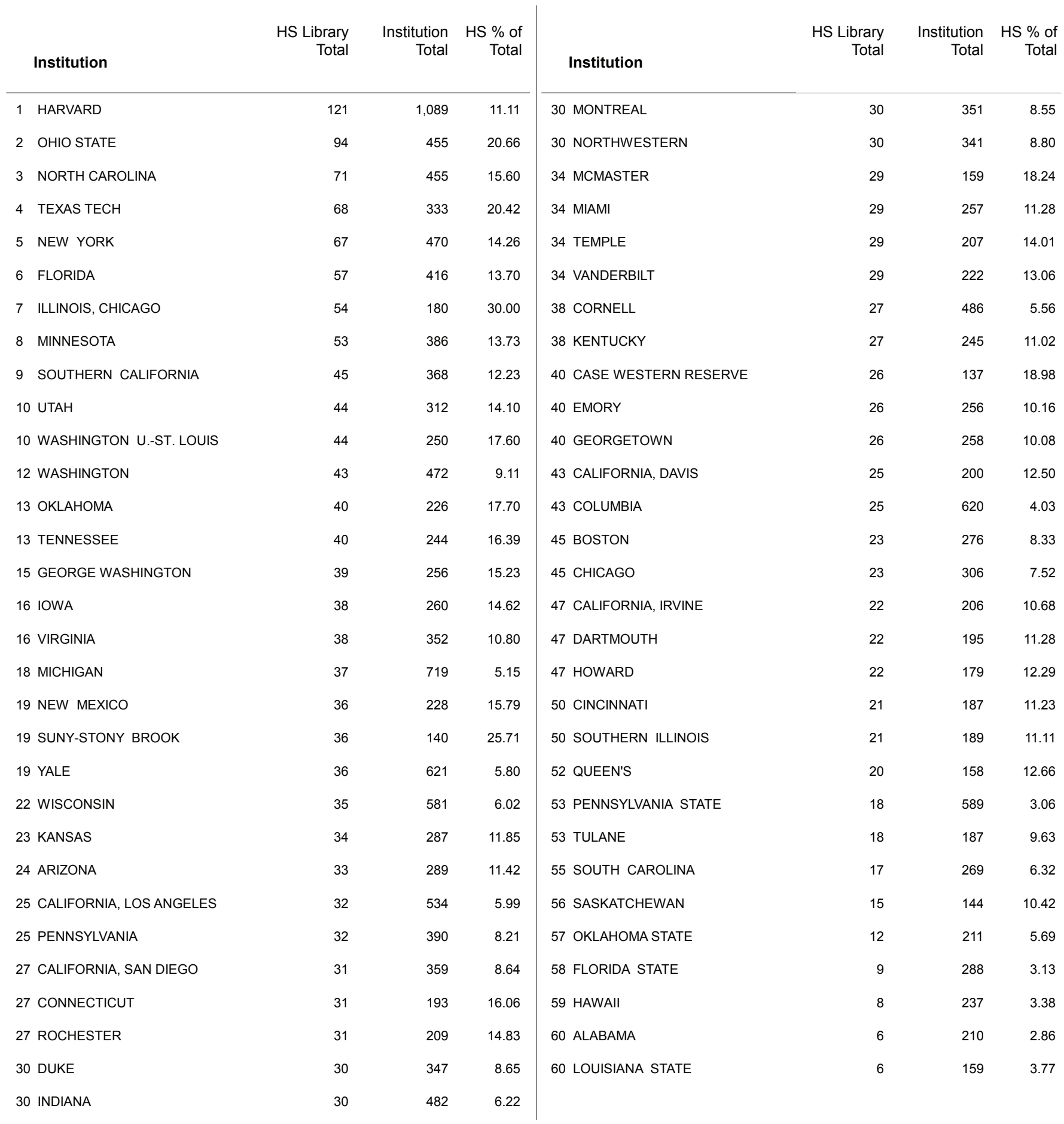


Rank Order Table 6: Total Electronic Resources Expenditures

\begin{tabular}{|c|c|c|c|c|c|c|c|c|}
\hline 2 & WASHINGTON U.-ST. LOUIS & $3,187,175$ & $7,163,364$ & 44.49 & 33 WISCONSIN & $1,433,304$ & $7,081,468$ & 20.24 \\
\hline 3 & IOWA & $2,858,904$ & $8,239,018$ & 34.70 & 34 MCMASTER & $1,423,730$ & $5,839,269$ & 24.38 \\
\hline 5 & WASHINGTON & $2,624,569$ & $8,581,484$ & 30.58 & 36 CINCINNATI & $1,380,211$ & $5,933,480$ & 23.26 \\
\hline 6 & MINNESOTA & $2,559,788$ & $9,797,966$ & 26.13 & 37 PENNSYLVANIA STATE & $1,367,657$ & $11,404,651$ & 11.99 \\
\hline 7 & SOUTHERN CALIFORNIA & $2,557,381$ & $10,790,439$ & 23.70 & 38 ARIZONA & $1,324,651$ & $8,555,435$ & 15.48 \\
\hline 8 & HARVARD & $2,385,093$ & $9,335,310$ & 25.55 & 39 OHIO STATE & $1,294,966$ & $7,191,692$ & 18.01 \\
\hline 11 & YALE & $2,224,819$ & $8,299,701$ & 26.81 & 42 CASE WESTERN RESERVE & $1,243,666$ & $3,943,642$ & 31.54 \\
\hline 12 & 2 GEORGE WASHINGTON & $2,123,920$ & $6,006,145$ & 35.36 & 43 BOSTON & $1,172,388$ & $6,428,024$ & 18.24 \\
\hline 13 & 3 TEMPLE & $2,109,284$ & $8,341,920$ & 25.29 & 44 DUKE & $1,152,179$ & $8,382,329$ & 13.75 \\
\hline 14 & 4 EMORY & $2,039,325$ & $6,927,350$ & 29.44 & 45 HOWARD & $1,119,796$ & $2,202,331$ & 50.85 \\
\hline 15 & 5 CONNECTICUT & $2,028,392$ & $7,494,542$ & 27.06 & 46 DARTMOUTH & $1,047,086$ & $6,532,195$ & 16.03 \\
\hline 16 & NORTHWESTERN & $1,966,291$ & $8,621,986$ & 22.81 & 47 CALIFORNIA, DAVIS & $1,041,423$ & $4,611,119$ & 22.59 \\
\hline 17 & 7 COLUMBIA & $1,959,627$ & $13,183,416$ & 14.86 & 48 NEW MEXICO & $1,001,552$ & $4,990,803$ & 20.07 \\
\hline 18 & 3 NEW YORK & $1,904,881$ & $12,112,955$ & 15.73 & 49 FLORIDA STATE & 899,258 & $6,642,481$ & 13.54 \\
\hline 24 & 4 KENTUCKY & $1,713,695$ & $6,867,494$ & 24.95 & 55 LOUISIANA STATE & 371,915 & $4,142,185$ & 8.98 \\
\hline 25 & 5 ILLINOIS, CHICAGO & $1,677,037$ & $7,757,472$ & 21.62 & 56 OKLAHOMA STATE & 191,833 & & \\
\hline 26 & OKLAHOMA & $1,669,848$ & $5,440,310$ & 30.69 & 57 SOUTHERN ILLINOIS & 143,343 & $4,124,280$ & 3.48 \\
\hline 27 & 7 FLORIDA & $1,576,985$ & $8,694,125$ & 18.14 & 58 ALABAMA & 83,285 & $5,094,382$ & 1.63 \\
\hline 28 & 3 QUEEN'S & $1,484,944$ & $5,967,600$ & 24.88 & CALIFORNIA, LOS ANGELES & . & $5,249,638$ & \\
\hline 29 & 9 SUNY-STONY BROOK & $1,462,438$ & $4,232,424$ & 34.55 & NORTH CAROLINA & & $7,046,460$ & \\
\hline 30 & CALIFORNIA, SAN DIEGO & $1,456,746$ & $5,661,298$ & 25.73 & SASKATCHEWAN & . & $5,728,897$ & \\
\hline 31 & 1 VIRGINIA & $1,438,726$ & $5,893,290$ & 24.41 & & & & \\
\hline
\end{tabular}




\section{ARL Statistics Questionnaire 2009-2010}

\section{Instructions for COMPLeting The QuestionnAire}

\section{General Instructions}

Definitions of statistical categories can be found in NISO Z39.7-2004, Information Services and Use: Metrics \& statistics for libraries and information providers--Data Dictionary (http://www.niso.org/). ARL has been modifying the interpretation of the standard definitions to address questions posed by library staff at various member institutions that complete the survey and with feedback from the ARL Statistics and Assessment Committee (http://www.arl.org/stats/aboutstats/index.shtml).

Please do not use decimals. All figures should be rounded to the nearest whole number.

Please respond to every question. If an exact figure cannot be provided, use NA/UA to indicate that the figure is either unavailable or not applicable. If the appropriate answer is zero or none, use $\mathbf{0 .}$

Although the form allows for data to be entered from both main and branch campuses, an effort should be made to report figures for the main campus only. (The U.S. National Center for Education Statistics, Integrated Postsecondary Education Data System (IPEDS) defines a branch institution as "a campus or site of an educational institution that is not temporary, is located in a community beyond a reasonable commuting distance from its parent institution, and offers organized programs of study, not just courses"). If figures for libraries located at branch campuses are reported, please specify which branch libraries are included and which ones are excluded in the notes below.

A branch library is defined as an auxiliary library service outlet with quarters separate from the central library of an institution, which has a basic collection of books and other materials, a regular staffing level, and an established schedule. A branch library is administered either by the central library or (as in the case of some law and medical libraries) through the administrative structure of other units within the university. Departmental study/reading rooms are not included.

The questionnaire assumes a fiscal year ending June 30, 2010. If your fiscal year is different, please indicate this in the notes below by adjusting the reporting period.

Footnotes. Explanatory footnotes will be included with the published statistics. Provide any notes you may have in the footnotes area at the end of the survey. Reporting libraries are urged to record there any information that would clarify the figures submitted in that line, e.g., the inclusion and exclusion of branch campus libraries. Please make an effort to word your footnotes in a manner consistent with notes appearing in the published report, so that the ARL Office can interpret your footnotes correctly. Please use a concise sentence/paragraph format when writing footnotes - do not use "bullets" or make a "bullet list."

\section{$\underline{\text { Specific Instructions }}$}

\section{Questions 1-1b. Volumes:}

Question 1. Volumes in Library. Use the ANSI/NISO Z39.7-2004 definition for volume as follows:

a single physical unit of any printed, typewritten, handwritten, mimeographed, or processed work, distinguished from other units by a separate binding, encasement, portfolio, or other clear distinction, which has been cataloged, classified, and made ready for use, and which is typically the unit used to charge circulation transactions. Either a serial volume is bound, or it comprises the serial issues that would be bound together if the library bound all serials. 
Include duplicates and bound volumes of periodicals. For purposes of this questionnaire, unclassified bound serials arranged in alphabetical order are considered classified. Exclude microforms, maps, nonprint materials, and uncataloged items. If any of these items cannot be excluded, please provide an explanatory footnote

Include government document volumes that are accessible through the library's catalogs regardless of whether they are separately shelved. "Classified" includes documents arranged by Superintendent of Documents, CODOC, or similar numbers. "Cataloged" includes documents for which records are provided by the library or downloaded from other sources into the library's card or online catalogs. Documents should, to the extent possible, be counted as they would if they were in bound volumes (e.g., 12 issues of an annual serial would be one or two volumes). Title and piece counts should not be considered the same as volume counts. If a volume count has not been kept, it may be estimated through sampling a representative group of title records and determining the corresponding number of volumes, then extrapolating to the rest of the collection. As an alternative, an estimate may be made using the following formulae:

\section{2 documents pieces per foot \\ 10 "traditional" volumes per foot \\ 5.2 documents pieces per volume}

Include e-book units, as long as these e-books are owned or leased and have been cataloged by your library. Include electronic books purchased through vendors such as NetLibrary ${ }^{\circledR}$ or Books $24 \times 7$, and e-books that come as part of aggregate services. Include individual titles of e-book sets that are treated as individual reference sources. Include locally digitized electronic books and electronic theses and dissertations. Provide a footnote explaining how many e-books you are reporting, preferably by specifying the products and the number of titles in a note.

Include volumes purchased collectively where the cost is shared at the time of purchase.

If either formulas or sampling are used for deriving your count, please indicate in a footnote.

Question 1b. Volumes Added. Include only volumes cataloged, classified, and made ready for use. Include government documents if they have been included in the count of volumes on line 1a. Do not include as part of Volumes Added Gross any government documents or other collections (such as large gift collections or e-book packages, EBBO, etc.) that were added to the collection as the result of a one time download or addition to the OPAC. Include these items in Volumes Held of the previous year (Line 1a) and provide a footnote explaining the revision of Line 1a.

Question 2. Titles Held. Use the ANSI/NISO Z39.7-2004 definition for title as follows:

The designation of a separate bibliographic whole, whether issued in one or several volumes .... Titles are defined according to the Anglo-American Cataloging Rules. A book or serial title may be distinguished from other such titles by its unique International Standard Book Number (ISBN) or International Standard Serial Number (ISSN). This definition applies equally to print, audiovisual, and other library materials. For unpublished works, the term is used to designate a manuscript collection or an archival record series. Two subscriptions to Science magazine, for example, are counted as one title. When vertical file materials are counted, a file folder is considered a title.

Report the total number of unique titles cataloged, classified and made ready for use. The number of titles reported here is for the number of volumes reported under line (1). Include e-books as specified above in question (1). For those reporting a bibliographic volume under line (1), their title count may be exactly the same as their volume count. 
Question 3. Monographic Volumes Purchased. Report number of volumes purchased; do not include volumes received or cataloged. Include all volumes for which an expenditure was made during 2009-10, including volumes paid for in advance but not received during the fiscal year. Include monographs in series and continuations. Include e-books that fit the NetLibrary ${ }^{\circledR}$ model, i.e., electronic manifestations of physical entities and/or units; provide a footnote explaining how many e-books you are reporting, preferably by specifying the products and the number of titles. If only number of titles purchased can be reported, please report the data and provide an explanatory footnote.

Question 4: Basis of Volume Count. A physical count is a piece count; a bibliographic count is a catalog record count.

Questions 5. Serials. Use the following definition adapted from AACR2 for a serial:
A bibliographic resource issued in a succession of discrete parts, usually bearing numbering, that has no predetermined conclusion. Examples of serials include journals, magazines, electronic journals, continuing directories, annual reports, newspapers, and monographic series.

Report the total number of unique serial titles, NOT SUBSCRIPTIONS, that you currently acquire and to which you provide access. Do not include duplicate counts of serial titles. Report each title once, regardless of how many subscriptions or means of access you provide for that title. Exclude unnumbered monographic and publishers' series. Electronic serials acquired as part of a bundle or an aggregated package should be counted at the title level, even if they are not cataloged, as long as the title is made accessible directly by the library (e.g., through a finding aid). If access is provided only through the overall platform or aggregator, do not report the individual titles but count the package as a single title.

Question 5a. Serial titles currently purchased. In the case of consortial agreements, count under 'serial titles currently purchased' those titles for which the library pays any amount from its budgeted expenditures. Include all titles that are part of bundles or aggregated packages, even if your library makes a partial payment for access to those titles. If a purchased title includes electronic access to the title, count that title ONLY ONCE (DEDUPED) as electronic only. If a database includes full-text and abstracted titles, the number of full-text titles can be counted.

Question 5b. Serial titles: Not Purchased. Report other titles that your library receives and does not pay for directly under 'serial titles received but not purchased.' These titles may include exchanges, gifts, etc.

If serial titles have been purchased through a consortium whose budget is centrally funded and independent from the library's budget, these serials should be reported under 'serial titles currently received but not purchased.' If within a purchased or aggregated package it cannot be determined that some titles are not purchased, report all titles as purchased.

Freely accessible titles are those your library provides direct access to via cataloging records or through online serial lists of other finding aids.

To the extent possible, report all government document serials separately in (5b.iv).

If separate counts of non-purchased and purchased serial titles are not available, report only the total number of serial titles currently purchased and received on line (5), and report NA/UA for lines (5a) and (5b).

Question 7. Microforms. Report the total number of physical units: reels of microfilm, microcards, and microprint and microfiche sheets. Include all government documents in microform; provide a footnote if documents are excluded. 
Question 8. Government documents. Report the total number of physical units (pieces) of government documents in paper format that have not been counted elsewhere. Include local, state, national, and international documents; include documents purchased from a commercial source if shelved with separate documents collections and not counted above. Include serials and monographs. To estimate pieces from a measurement of linear feet, use the formula 1 foot $=52$ pieces and indicate in a footnote that the count is based on this estimate. Exclude microforms and non-print formats such as maps or CD-ROMs. Adjust line (1a), i.e., last year's Volumes Held, and provide a footnote if you are adding records to the OPAC for government documents previously held but not counted as part of Volumes Held line (1a).

Question 9. Computer files. Include the number of pieces of computer-readable disks, tapes, CD-ROMs, and similar machinereadable files comprising data or programs that are locally held as part of the library's collections available to library clients. Examples are U.S. Census data tapes, sample research software, locally-mounted databases, and reference tools on CD-ROM, tape or disk. Exclude bibliographic records used to manage the collection (i.e., the library's own catalog in machine-readable form), library system software, and microcomputer software used only by the library staff.

Question 10. Manuscripts and archives. Include both manuscripts and archives measured in linear feet.

Question 11. Cartographic materials. Include the numbers of pieces of two- and three-dimensional maps and globes. Include satellite and aerial photographs and images.

Question 12. Graphic materials. Include the number of pieces of prints, pictures, photographs, postcards, slides, transparencies, film strips, and the like.

Question 13. Audio materials. Include the number of pieces of audiocassettes, phonographic discs, audio compact discs, reel-toreel tapes, and other sound recordings.

Question 14. Film and video materials. Include the number of pieces of motion pictures, videocassettes, video laser discs, and similar visual materials.

Questions 15-21. Expenditures. Report all expenditures of funds that come to the library from the regular institutional budget, and from sources such as research grants, special projects, gifts and endowments, and fees for service. (For question (18), include nonlibrary funds; see instruction for question (18). Do not report encumbrances of funds that have not yet been expended. Canadian libraries should report expenditures in Canadian dollars. (For your information, if interested in determining figures in U.S. dollars, divide Canadian dollar amounts by 1.0556, the average monthly noon exchange rate published in the Bank of Canada Review for the period July 2009-June 2010). Please round figures to the nearest dollar.

Question 16a. Monographs. Report expenditures for volumes purchased counted on line (3).

Question 16b. Serial titles. Report expenditures for serial titles counted on line (5a). Exclude unnumbered monographic and publishers' series, and encumbrances.

Question 16c. Other library materials. Include expenditures for all materials not reported in Questions (16a) and (16b), e.g., backfiles of serials, charts and maps, audiovisual materials, manuscripts, etc. If expenditures for these materials are included in lines (16a) and/or (16b) and cannot be disaggregated, please report U/A and provide a footnote. Do not include encumbrances.

Question 16d. Miscellaneous expenditures. Include any other materials funds expenditures not included in questions (16a)-(16c), e.g., expenditures for bibliographic utilities, literature searching, security devices, memberships for the purposes of publications, etc. Please list categories, with amounts, in a footnote. Note: If your library does not use materials funds for non- 
materials expenditures - i.e., if those expenditures are included in "Other Operating Expenditures" - report 0, not NA/UA, on line (16d).

Question 17. Contract Binding. Include only contract expenditures for binding done outside the library. If all binding is done inhouse, state this fact and give in-house expenditures in a footnote; do not include personnel expenditures.

Questions 18. Salaries and wages. Exclude fringe benefits. If professional, support staff and student salaries cannot be separated, enter NA/UA, in lines (18a), (18b) and (18c) and enter total staff salaries in line (18).

Question 18c. Salaries and wages: Student Assistants. Report 100\% of student wages regardless of budgetary source of funds. Include federal and local funds for work study students.

Question 20. Other operating expenditures. Exclude expenditures for buildings, maintenance, and fringe benefits.

Questions 22-26. Electronic expenditures. These items are intended to indicate what portion of your institution's total library expenditures are dedicated to electronic resources and services. Please use the Footnotes to indicate any electronic materials expenditures you believe not to be covered by these questions. Many expenditures recorded in these questions should have been included in question (21), total library expenditures.

Question 22. One-time electronic resource purchases. Report expenditures that are not current serials (i.e. are non-subscription, one-time, or monographic in nature) for software and machine-readable materials considered part of the collections. Examples include periodical backfiles, literature collections, one-time costs for JSTOR membership, etc. Expenditures reported here may be derived from any of the following categories: Monographs (16a), Other Library Materials (16c), Miscellaneous (16d), or Other Operating Expenditures (20).

Question 23. Ongoing electronic resource purchases. Report subscription expenditures (or those which are expected to be ongoing commitments) for serial publications whose primary format is electronic and for online searches of remote databases such as OCLC FirstSearch, DIALOG, Lexis-Nexis, etc. Examples include paid subscriptions for electronic journals and indexes/abstracts available via the Internet, CD-ROM serials, and annual access fees for resources purchased on a "one-time" basis, such as literature collections, JSTOR membership, etc. Not all items whose expenditures are counted here will be included in Serial titles currently received question (5) or Serials Expenditures question (16b).

Question 24. Bibliographic Utilities, Networks, and Consortia. Because it is increasingly common for ARL Libraries to enter into consortial arrangements to purchase access to electronic resources, both "Library" and "External" expenditure blanks and instructions are provided. Please use afootnote to describe expenditures that you believe are not covered by the question, or situations that do not seem to fit the instructions.

Question 24a. From internal library sources. Report expenditures paid by the Library for services provided by national, regional, and local bibliographic utilities, networks, and consortia, such as OCLC and RLG, unless for user database access and subscriptions, which should be reported in questions (22) or (23). Include only expenditures that are part of Other Operating Expenditures (Q20).

Question 24b. From external sources. If your library receives access to computer files, electronic serials or search services through one or more centrally-funded system or consortial arrangements for which it does not pay fully and/or directly (for example, funding is provided by the state on behalf of all members), enter the amount paid by external bodies on its behalf. If the specific dollar amount is not known, but the total student FTE for the consortium and amount spent for the academic members are known, divide the overall amount spent by your institution's share of the total student FTE. 
Question 25. Computer hardware and software. Report expenditures from the library budget for computer hardware and software used to support library operations, whether purchased or leased, mainframe or microcomputer, and whether for staff or public use. Include expenditures for: maintenance; equipment used to run information service products when those expenditures can be separated from the price of the product; telecommunications infrastructure costs, such as wiring, hubs, routers, etc. Include only expenditures that are part of Other Operating Expenditures (20).

Question 26. Document Delivery/Interlibrary Loan. Report expenditures for document delivery and interlibrary loan services (both borrowing and lending). Include fees paid for photocopies, costs of telefacsimile transmission, royalties and access fees paid to provide document delivery or interlibrary loan. Include fees paid to bibliographic utilities if the portion paid for interlibrary loan can be separately counted. Include only expenditures that are part of Miscellaneous Materials Expenditures (16d) or Other Operating Expenditures (20), and only for those ILL/DD programs with data recorded in Questions (35)-(36).

Questions 27. Personnel. Report the number of FTE (full-time equivalent) staff in filled positions, or positions that are only temporarily vacant. ARL defines temporarily vacant positions as positions that were vacated during the fiscal year for which ARL data were submitted, for which there is a firm intent to refill, and for which there are expenditures for salaries reported on line (18).

Include cost recovery positions and staff hired for special projects and grants, but provide an explanatory footnote indicating the number of such staff. If such staff cannot be included, provide a footnote. To compute full-time equivalents of part-time employees and student assistants, take the total number of hours per week (or year) worked by part-time employees in each category and divide it by the number of hours considered by the reporting library to be a full-time work week (or year). Round figures to the nearest whole numbers.

Question 27a. Professional Staff. Since the criteria for determining professional status vary among libraries, there is no attempt to define the term "professional." Each library should report those staff members it considers professional, including, when appropriate, staff who are not librarians in the strict sense of the term, for example computer experts, systems analysts, or budget officers.

Question 27b. Support Staff. Report the total FTE (see instruction (27) of staff not included in (27a).

Question 27c. Student Assistants. Report the total FTE (see instruction Q27) of student assistants employed on an hourly basis whose wages are paid from funds under library control or from a budget other than the library's, including federal work-study programs. Exclude maintenance and custodial staff.

Question 28. Number of staffed library service points. Count the number of staffed public service points in the main library and in all branch libraries reported in this inventory, including reference desks, information desks, circulation, current periodicals, reserve rooms, reprographic services (if staffed as a public facility), etc. Report the number of designated locations, not the number of staff.

Question 29. Number of weekly public service hours. Report an unduplicated count of the total public service hours per typical full-service week (i.e., no holidays or other special accommodations) across both main library and branches using the following method (corresponds to IPEDS): If a library is open from 9:00 a.m. to 5:00 p.m. Monday through Friday, it should report 40 hours per week. If several of its branches are also open during these hours, the figure remains 40 hours per week. Should Branch A also be open one evening from 7:00 p.m. to 9:00 p.m., the total hours during which users can find service somewhere within the system becomes 42 hours per week. If Branch B is open the same hours on the same evening, the count is still 42, but if Branch B is open two hours on another evening, or remains open two hours later, the total is then 44 hours per week. Exclude 24-hour unstaffed reserve or similar reading rooms. The maximum total is 168 (i.e., a staffed reading room open 7 days per week, 24 hours per day).

Questions 30-31. Instruction. Sampling based on a typical week may be used to extrapolate TO A FULL YEAR for Questions (30) and (31). Please indicate if responses are based on sampling. 
Question 30. Presentations to Groups. Report the total number of sessions during the year of presentations made as part of formal bibliographic instruction programs and through other planned class presentations, orientation sessions, and tours. If the library sponsors multi-session or credit courses that meet several times over the course of a semester, each session should be counted. Presentations to groups may be for either bibliographic instruction, cultural, recreational, or educational purposes. Presentations both on and off the premises should be included as long as they are sponsored by the library. Do not include meetings sponsored by other groups using library meeting rooms. Do not include training for library staff; the purpose of this question is to capture information about the services the library provides for its clientele. Please indicate if the figure is based on sampling.

Question 31. Participants in Group Presentations. Report the total number of participants in the presentations reported on line (30). For multi-session classes with a constant enrollment, count each person only once. Personal, one-to-one instruction in the use of sources should be counted as reference transactions on line (32). Please indicate if the figure is based on sampling. Use a footnote to describe any special situations.

Question 32. Reference Transactions. Report the total number of reference transactions. A reference transaction is an information contact that involves the knowledge, use, recommendations, interpretation, or instruction in the use of one or more information sources by a member of the library staff. The term includes information and referral service. Information sources include (a) printed and nonprinted material; (b) machine-readable databases (including computer-assisted instruction); (c) the library's own catalogs and other holdings records; (d) other libraries and institutions through communication or referral; and (e) persons both inside and outside the library. When a staff member uses information gained from previous use of information sources to answer a question, the transaction is reported as a reference transaction even if the source is not consulted again.

If a contact includes both reference and directional services, it should be reported as one reference transaction. Include virtual reference transactions (e.g., e-mail, WWW form, chat). Duration should not be an element in determining whether a transaction is a reference transaction. Sampling based on a typical week may be used to extrapolate TO A FULL YEAR for Question 32. Please indicate if the figure is based on sampling.

EXCLUDE SIMPLE DIRECTIONAL QUESTIONS. A directional transaction is an information contact that facilitates the logistical use of the library and that does not involve the knowledge, use, recommendations, interpretation, or instruction in the use of any information sources other than those that describe the library, such as schedules, floor plans, and handbooks.

Questions 33-34. Circulation. For Question (33), count the number of initial circulations during the fiscal year from the general collection for use usually (although not always) outside the library. Do not count renewals. Include circulations to and from remote storage facilities for library users (i.e., do not include transactions reflecting transfers or stages of technical processing). Count the total number of items lent, not the number of borrowers.

For Question (34), report total circulation for the fiscal year including initial transactions reported on line (33) and renewal transactions. Exclude reserve circulations; these are no longer reported.

Questions 35-36. Interlibrary Loans. Report the number of requests for material (both returnables and non-returnables) provided to other libraries on line (35) and the number of filled requests received from other libraries or providers on line (36). On both lines, include originals, photocopies, and materials sent by telefacsimile or other forms of electronic transmission. Include patron-initiated transactions. Exclude requests for materials locally owned and available on the shelves or electronically. Do not include transactions between libraries covered by this questionnaire. 
Question 37. PhD Degrees. Report the number awarded during the 2007-08 fiscal year. Please note that only the number of $\underline{\mathrm{Ph} . \mathrm{D}}$. degrees are to be counted. Statistics on all other advanced degrees (e.g., D.Ed., D.P.A., M.D., J.D.) should not be reported in this survey. If you are unable to provide a figure for Ph.D.s only, please add a footnote.

Question 38. PhD Fields. For the purposes of this report, Ph.D. fields are defined as the specific discipline specialties enumerated in the U.S. Department of Education's Integrated Postsecondary Education Data System (IPEDS) “Completions" Survey. Although the IPEDS form requests figures for all doctoral degrees, only fields in which $\underline{\mathrm{PhDs}}$ are awarded should be reported on the ARL questionnaire. Any exceptions should be footnoted.

Question 39. Instructional Faculty. Instructional faculty are defined by the U.S. Dept. of Education as:

members of the instruction/research staff who are employed full-time as defined by the institution, including faculty with released time for research and faculty on sabbatical leave.

Full-time counts generally exclude faculty who are employed to teach fewer than two semesters, three quarters, two trimesters, or two four-month sessions; replacements for faculty on sabbatical leave or leave without pay; faculty for preclinical and clinical medicine; faculty who are donating their services; faculty who are members of military organizations and paid on a different pay scale from civilian employees; academic officers, whose primary duties are administrative; and graduate students who assist in the instruction of courses. Please be sure the number reported, and the basis for counting, are consistent with those for 2006-07 (unless in previous years faculty were counted who should have been excluded according to the above definition). Please footnote any discrepancies.

Questions 40-43. Enrollment. U.S. libraries should use the Fall 2009 enrollment figures reported to the Department of Education on the Integrated Postsecondary Education Data System survey. Please check these figures against the enrollment figures reported to ARL last year to ensure consistency and accuracy. Note: In the past, the number of part-time students reported was FTE; the number now reported to IPEDS is a head count of part-time students. Canadian libraries should note that the category "graduate students" as reported here includes all post-baccalaureate students.

\section{FOOTNOTES}

Please consult the data entry Web interface (www.arlstatistics.org) for a copy of last year's footnotes. These can be found under "Data Repository" after you login into www.arlstatistics.org. Explanatory footnotes will be included with the published statistics. Reporting libraries are urged to record in the footnote section any information that would clarify the figures submitted, e.g., the inclusion and exclusion of branch campus libraries (see the "General Instructions" for definition of branch campus libraries). Please make an effort to word your footnotes in a manner consistent with notes appearing in the published report, so that the ARL Office can interpret your footnotes correctly.

NOTE: Any change over 10\% in any answer to any of the survey's questions over the preceding year's response (2008-09) should be addressed with a footnote.

Submit the completed questionnaire by

October 15, 2010.

For assistance, please e-mail Martha Kyrillidou (martha@arl.org) or Shaneka Morris (shaneka@arl.org)

Tel. (202) 296-2296. 


\section{ARL Statistics WorksheEt 2009-2010}

This worksheet is designed to help you plan your submission for the 2009-2010 ARL Statistics. The figures on this worksheet should be similar to those in the "Summary" page of your web form, except in cases where data are unavailable. If an exact figure is unavailable, use "NA/UA". If the appropriate answer is zero or none, use " 0. "

Reporting Institution Date Returned to ARL

Report Prepared by (name)

Title

Email address

Phone number

Contact person (if different)

Title

Email address Phone number

PAGE ONE - VOLUMES AND TITLES:

1. Volumes held June 30, $2010(1 . a+1 . b)$

(1)

1a. Volumes held June 30, 2009

(1.a)

1b. Volumes added during the year (1.b.i - 1.b.ii)

(i) Volumes added - Gross

(ii) Volumes withdrawn during year

(1.b.ii)

2. Titles held June 30, 2010

(2)

3. Number of monographic volumes purchased

(3)

4. Basis of volume count is:

(4)

Physical

Bibliographic 
PAGE TWO - OTHER COLLECTIONS

SERIALS

5. Total number of serial titles currently received, including periodicals $(5 . a+5 . b)$

(5)

5a. Number of serial titles currently purchased $(5 a . i+5 a . i i)(5 a)$

5a.i Electronic

5a.ii Print (and other format) serials purchased

(5a.ii)

$5 b$. Number of serial titles currently received but not purchased

$$
(5 b . i+5 b . i i+5 b . i i i+5 b . i v)
$$

5b.i Consortial

5b.ii Freely accessible

5b.iii Print (and other format) - Exchanges,

(5b.iii) gifts, etc.

5b.iv Government documents

(5b.iv)

6. Government documents are included in count of Current Serials?

(6) Yes No

\section{OTHER LIBRARYMATERIALS}

\section{Microform units}

(7)

8. Government documents not counted elsewhere

(8)

9. Computer files

(9)

10. Manuscripts and archives (linear ft.)

(10)

\section{AUDIOVISUAL MATERIALS}

11. Cartographic

12. Graphic

13. Audio

14. Film and Video 
PAGE THREE - EXPENDITURES

15. Are the below figures reported in Canadian dollars?

(15) Yes

No

16. Total Library Materials Expenditures $(16 \cdot a+16 . b+16 . c+16 . d)$

(16)
16a. Monographs
(16a)
16b. Serial titles, including periodicals
(16b)
16c. Other Library Materials
(16c)
16d. Miscellaneous
(16d)

17. Contract binding

(17)

18. Total Salaries and Wages $(18 . a+18 . b+18 . c)$

(18)

18a. Professional staff

(18a)

18b. Support staff

18c. Student assistants

(18c)

19. Fringe benefits are included in expenditures for salaries and wages? (19) Yes No

20. Other operating expenditures

(20)

21. Total library expenditures $\quad(16+17+18+20)$

(21)

\section{ELECTRONIC MATERIALS EXPENDITURES}

22. One-time electronic resource purchases

23. Ongoing electronic resource purchases (e.g., subscriptions, annual license fees)

(23)

24. Bibliographic Utilities, Networks, and Consortia

24a. From internal library sources

(24a)

24b. From external sources

25. Computer hardware and software

(25)

26. Document Delivery/Interlibrary Loan

(26) 
PAGE FOUR - PERSONNEL AND PUBLIC SERVICES

PERSONNEL (Round figures to nearest whole number.)

27. Total Staff FTE $(27 . a+27 . b+27 . c)$

27a. Professional staff, FTE

(27a)

27b. Support staff, FTE

(27b)

27c. Student assistants, FTE

$(27 \mathrm{c})$

\section{STAFFED SERVICE POINTS AND HOURS}

28. Number of staffed library service points

(28)

29. Number of weekly public service hours

(29)

\section{INSTRUCTION}

30. Number of library presentations to groups

(30)

30a. Is the library presentations figure based on sampling?

(30a) Yes No

31. Number of total participants in group presentations reported in line 30

31a. Is the total participants in group presentations figure based on sampling?

(31a) Yes No

\section{REFERENCE}

32. Number of reference transactions

(32)

32a. Is the reference transactions figure based on sampling?

(32a) Yes No 


\section{CIRCULATION}

33. Number of initial circulations (excluding reserves)

34. Total circulations (initial and renewals, excluding reserves)

\section{INTERLIBRARYLOANS}

\section{Total number of filled requests provided to other libraries}

36. Total number of filled requests received from other libraries or providers

\section{PhD DEGREES AND FACULTY}

37. Number of PhDs awarded in FY2008-2009

38. Number of fields in which PhDs can be awarded

39. Number of full-time instructional faculty in FY2008-2009

ENROLLMENT - FALL 2008

(Line numbers refer to IPEDS survey form.)

40. Full-time students, undergraduate and graduate

(Add line 8, columns $15 \mathcal{E} 16$, and line 14, columns $15 \mathcal{E} 16$. )

41. Part-time students, undergraduate and graduate

(Add line 22, columns $15 \& 16$, and line 28, columns 15 \& 16.)

43. Part-time graduate students (Line 28, columns $15 \mathcal{E} 16$.

\section{FOOTNOTES}

On the web form, you will be able to add footnotes to individual questions, as well as footnotes that apply to your entire institution. Please provide any information which would clarify the figures submitted, e.g., the inclusion of branch campus libraries or any special projects which might cause radical increases or decreases. Please use the footnotes in the ARL Statistics 2008-2009 for comparison if necessary. Please consult the Data Repository under www.arlstatistics.org for a copy of last year's footnotes. These can be found under "Data Repository" after you login to www.arlstatistics.org. Please make an effort to word your footnotes in a manner consistent with notes appearing in the published report, so that the ARL Office can interpret your footnotes correctly. Please use a concise sentence/paragraph format when writing footnotes-do not use bullets or make a bullet list.

NOTE: Any change over 10\% in any answer to any of the survey's questions over the preceding year's response (2008-09) should be addressed with a footnote.

Submit the completed questionnaire by

October 15, 2010.

For assistance, please e-mail Martha Kyrillidou (martha@arl.org) or Shaneka Morris (shaneka@arl.org) Tel. (202) 296-2296. 


\section{FOOTNOTES}

Footnotes may also include errata and corrections to data not previously reported from prior years. Numbers refer to columns in Library Data Tables and to Questionnaire numbers. Unless otherwise noted, all figures are as of 06/30/2010.

QUESTION FOOTNOTE

NUMBER

ALABAMA

All figures are as of 09/30/2010.

5.b.i We do not currently have any way of separating "Consortial" and "Freely Accessible Titles." Therefore, they are all recorded here as "Freely Accessible."

ARIZONA

1.a Volumes held June 30, 2009 revised to 230,975.

12, 13 Estimated - extensive discarding during 2010.

\section{CALIFORNIA, DAVIS}

1.b, 1.b.i Added fewer items, did not buy as many titles.

3 Bought fewer monographs than in 2008-2009.

5, 5.a, Increase due to better reporting from SFX and the inclusion of free or open access titles we provide through SFX.

$5 . b$

Incorrect value entered in 2008-2009. Correct value is 12,769 .

12

Incorrect value entered in 2008-2009. Correct value is 419.

13

The audio withdrawn represents the winnowing down of the Audio-Digest CD collection to just the 2009-2010 items plus the preceding two years.

Incorrect value entered in 2008-2009. Correct value is 259 .

16

Decrease due to reduced purchases during the year. Several of the licensed resources had reductions in the cost of the package for the calendar year 2010.

16.a This year's number is considerably lower because of one-time purchases the prior year.

17, $25 \quad$ Reported in Main Library Statistics.

18.a Expenditures for staffing are significantly less than in 2008-2009 because of retirements and vacant positions not being filled. Staff also experienced furloughs during part of the fiscal year which reduced their base pay.

18.c Expenditures for staffing are significantly less than in 2008-2009 because of vacant positions not being filled.

20 Incorrect value entered in 2008-2009. Moreover, insurance deductible was higher due to water damage claim in 2008-2009. Installation of computer and telephone connections for relocated staff also contributed to the increase.

33, 34 The 2009-2010 number represents a more accurate recording of the circulation numbers. However, it will include anomalies related to checking out materials to the library for inventory purposes and other similar projects.

The SERHOLD update completed during the year resulted in fewer requests coming to UC Davis.

36 Fewer requests received, perhaps as a result of an increased number of electronic resource packages.

\section{CALIFORNIA, IRVINE}

1.a Volumes held June 30, 2009 revised to 374,671.

\section{CALIFORNIA, LOS ANGELES}

1.a Volumes held June 30, 2009 revised to 669,755.

2 Previous year reported as the same count as in question 1, this year reflects actual count based on definition for unique titles. 
NUMBER

\section{CALIFORNIA, LOS ANGELES, cont}

There is no separate count for serials for Health Sciences, please see Main Library data for electronic and print serials.

\section{CALIFORNIA, SAN DIEGO}

Library branches included: Biomedical Center Library, Medical Center Library.

1.a

Volumes held June 30, 2009 revised to 240,718.

\section{CASE WESTERN RESERVE}

1.b.i

5.a.i

\section{CHICAGO}

1.b-1.b.ii

5.a.i-

5.a.ii

14

$16,16 . b-$

$16 . \mathrm{c}$

17

$18,18 . b-$

$18 . \mathrm{c}$

20

29

\section{CINCINNATI}

1.b, 1.b.ii Negative numbers reflect weeding of some bound journals in the Health Sciences Library.

Serials not reported separately from total university count.

This decrease from 2008-2009 reflects fewer print journals purchased and more careful attention to not binding when archival rights are held to an electronic journal.

\section{COLUMBIA}

5, $26 \quad$ Included in Butler Library statistics.

12 The Wilcox Collection.

\section{CORNELL}

1.a

Volumes held June 30, 2009 revised to 204,027 to reflect additional e-books. The increase in the count of e-books reflects both the availability of new titles and the availability of online records for previously held collections.

Includes 322 e-books cataloged manually. The library had an $80 \%$ budget cut for print monograph acquisitions. This, combined with more e-journals instead of print journals, caused the decrease in volumes added new. 
NUMBER

CORNELL, cont.

2

\section{DARTMOUTH}

Library branches included: Dana Biomedical Library, Matthews-Fuller Health Sciences Library.

1.b.i

\section{EMORY}

All figures are as of 08/31/2010.

Library branches included: Grady Branch, Emory Hospital Branch, Emory Midtown Branch (new and not full year; no volume holdings added to count).

1.b.ii Increase due to withdrawal of all bound journal volumes from a branch library, a total of 16,237 volumes discarded as

follows: 2,100 volumes to Nigerian library and 14,137 volumes to recycling.

The large increase reflects the fact that the count for 2008-2009 mistakenly excluded the e-book count (9,737 - some portion of which overlapped with the print). estimated to be non-current. The decrease in the print count reflects cancellations to favor electronic over print and budgetary pressures. The decrease in the number of titles estimated ceased is due to the use of a new methodology to estimate.

Count is for videos/DVDs.

Shipping costs only.

Binding was stopped as of July 2009 due to budgetary pressures. The library has very few print journal subscriptions.

Starting with 2009-2010, the amount for "professional" expenditures includes expenditures for academic and exempt staff. In the past, it included only expenditures for academic staff. expenditures for non-academic exempt and non-exempt staff.

Starting with 2009-2010, the "professional" staff count includes academic and exempt staff. In the past, it included academic staff only.

Starting with 2009-2010, this count includes only non-academic non-exempt staff. In the past, it included non-academic exempt and non-exempt staff.

Includes some instruction sessions for individuals.

Indicates emphasis on electronic-only and reduction due to budget constraints.

Withdrawals part of ongoing deaccessioning project.

Indicates emphasis on e-journals.

Did not include freely accessible e-journals with Health library figure, included with Main Library figure.

Transition to electronic journals from print journals.

Difficult to distinguish one library's electronic holdings from another library's especially when there is no payment, so included all freely accessible under the Main Library figure of 11,086.

Reflects emphasis on e-journals.

Reduction due to budget constraints.

Included with Main Library figure of $\$ 404,321$.

Reflects increase in use of our collection. 
NUMBER

\section{EMORY, cont.}

5.a.ii Decrease due to ongoing transition to electronic format serials.

16.a Increase due to more e-book purchases.

16.c Decrease due to spending less on backfile purchases than in 2008-2009.

17 Efforts to reduce contract binding are ongoing plus continuing transition to electronic format only for serials.

18.b, 27 Reduction in force took place in August 2009.

18.c Decrease due to having no student employees.

30 Increase due to implementation of spreadsheet used by all librarians and informationists to capture increased instruction detail.

32 Decrease due to fewer in-person questions and closure of Media service point.

\section{FLORIDA}

Library branches included: Borland Library, VetMed Reading Room.

\section{FLORIDA STATE}

16.a, 22 During 2008-2009, we acquired a number of e-books from OVID but did not do the same this year.

16.b This represents increases by publishers and efforts to receive the best pricing through multi-year plans.

16.c This represents savings realized by last year's multi-year plans.

18.b Due to an increase in support staff hired (see footnote for question 27b) there has been an overall increase in support staff funding.

The ILL traffic in 2009-2010 has increased (see questions 35 \& 36). This has resulted in an increase in ILL costs.

The professional librarian staff was down by two for the period from July 2008 to June 2009. One professional librarian was hired, accounting for the difference.

27.b One support staff member was added bringing up the total difference $50 \%$.

32 The 2008-2009 figures were based on sampling plus calculations. This year's method has changed; the data is not based on sampling and is therefore more accurate.

36 The overall enrollment has gone up, causing the ILL requests to go up.

\section{GEORGE WASHINGTON}

$1 . b$

2

4

5.a.i

5.b.ii

13

16.c

17

18.c

22

23
Number withdrawn in 2008-2009 was exceptionally large due to a physical space reduction. This year is a more typical number.

A large number of AV materials were weeded as well as titles.

At this time, both methods are used: physical counts for \#1 and bibliographic count accounts for \#2.

Converted many print+online titles to online-only allowing us to purchase additional titles.

University added Springer package, increasing the number.

Video tape collection weeded in anticipation of format change to DVD.

Electronic backfiles were purchased.

Binding has been eliminated.

Number of student workers used was modified.

More electronic backfiles were purchased than in 2008-2009.

Figure was calculated differently this year. 
NUMBER

\section{GEORGE WASHINGTON, cont.}

25 We purchased a large amount of computer hardware this year.

27

27.a

27.b

27.c

29

\section{GEORGETOWN}

1.a

Volumes held June 30, 2009 revised to 99,972.

\section{ILLINOIS, CHICAGO}

Library branches included: Library of the Health Sciences, Chicago; Library of the Health Sciences, Rockford; Library of the Health Sciences, Peoria; and Library of the Health Sciences, Urbana.

1.a Volumes held June 30, 2009 revised to 743,295.

16. The figure for total library materials expenditures for 2008-2009 was incorrect. The correct figure is for the 2008-2009 data $\$ 2,576,895$.

16.a

16.b

16.c

The figure for expenditures for monographs for 2008-2009 was incorrect. The correct figure for the 2008-2009 data is $\$ 403,306$.

The figure for expenditures for serials, including periodicals for 2008-2009 was incorrect. The correct figure for the 2008-2009 data is $\$ 2,128,932$.

The figure for expenditures for other library materials for 2008-2009 was incorrect. The correct figure for the 2008-2009 data is $\$ 44,657$.

\section{IOWA}

Volume count is up as the psych collection was incorporated when the psych library was closed.

\section{KANSAS}

Added many items that were on the shelves but not listed in the ongoing inventory.

Only reported monographs in 2008-2009.

No electronic monograph purchases in 2009-2010. Medical Library used resources purchased by the Lawrence campus library.

24.a Continued to use money from a prepaid credit account.

\section{KENTUCKY}

Total includes the following e-books: NetLibrary, 170; Methods in Enzymology, 13; Barash Clinical Anaesthesia, 1; Doody's 2009 Essential Collection, 55; Springer Collection on Medicine, 325; Stat!Ref, 50; and Ovid e-books, 4. Total represents print only; Medical Center Library e-serials are included in the total for the Main Library. Not able to provide a separate total for electronic serials currently purchased for the Medical Center Library. The total for the Main Library includes the Medical Center Library electronic serials. Not able to break out a separate total for the Medical Center Library. Number of consortial e-serials is included in the total for the Main Library.

5.b.ii Not able to provide separate total for the Medical Center Library. Freely accessible serial titles for the Medical Center Library are included in the total for the Main Library. 
NUMBER

\section{LOUISIANA STATE}

5, 5.a, Increase due to budgetary changes in priorities.

18.c, 27.c

16, 16.a, Decrease due to budget cuts.

20,27 ,

27.b

\section{MCMASTER}

All figures are as of 04/30/2010.

9

Major weeding project of computer files in 2009-2010.

$16-26$

Expenditures as reported in Canadian dollars: (16a) \$387,000; (16b) \$1,234,644; (16c) \$277,039; (16d) \$46,423; (16) \$1,945,106; (17) $\$ 788 ;$ (18a) \$567,085; (18b) \$804,451; (18c) \$77,697; (18) \$1,449,233; (20) \$96,005; (21) \$3,491,132; (22) \$46,980; (23) \$1,455,909; (24a) $\$ 25,000$; (24b) \$509; (25) \$35,466; (26) \$25,440.

MIAMI

All figures are as of 05/31/2010.

Figure includes audiovisual materials (questions 11 through 14) and computer files (question 9).

Excludes Medical Library Director's salary.

Includes library faculty only. Medical library reports all non-faculty professional staff in question 27b.

Includes both support and non-faculty professional staff.

Consultation sessions are not included, a change from prior year.

MINNESOTA

Library branches included: Bio-Medical Library, Veterinary Medical Library.

1.a

3

4

5.a, 5.b

12

24.a,

24. $b$

MONTREAL

All figures are as of 05/31/2010.

1.b

3

16

$16-26$

Print: 1,102; Electronic: 5,382. Expenditures overall.
Volumes held June 30, 2009 revised to 543,215.

Count of volumes purchased is not available. Count of monographs ordered is reported.

Based on count of item records not bibliographic records.

Not possible to separate and delineate count of unique titles by branch library.

Breakdown by format is not available. Total AV count for Health Sciences Libraries is 4,188.

Included with/paid by Main Library.

With the electronic books gaining popularity and place in our collection, we applied a strict weeding library plan.

Percent changes in excess of -10\% over the 2008-2009 figures for the sub parts of question 16 are due to the weeding work we have done in 2009-2010 in our physical collections. These decreases resulted in the decrease in Total Library Materials

Expenditures as reported in Canadian dollars: (16a) \$261,382; (16b) \$1,855,575; (16c) \$0; (16d) \$0; (16) \$2,116,957; (17) \$6,402; (18a) $\$ 735,828 ;$; (18b) \$1,063,194; (18c) \$0; (18) \$1,799,022; (20) \$19,870; (21) \$3,942,251; (22) \$178,930; (23) \$1,773,564; (24a) \$0; (24b) \$0; (25) $\$ 0 ;(26) \$ 0$. 
QUESTION FOOTNOTE

NUMBER

\section{NEW MEXICO}

1.a

16.c

20

NEW YORK

All figures are as of 08/31/2010.

\section{NORTH CAROLINA}

1.a

Volumes held June 30, 2009 revised to 347,025. Increased by 5,443 to reflect more accurate records now derived from online catalog.

12

Decrease reflects more accurate counts now derived from online catalog, plus withdrawal of several large fiche sets.

Replaced container counts with item counts.

\section{NORTHWESTERN}

All figures are as of 08/31/2010.

\section{OHIO STATE}

1.a $\quad$ Volumes held June 30, 2009 revised to 288,172.

\section{OKLAHOMA STATE}

33-34 We have no way to exclude reserves from this number.

\section{OKLAHOMA}

1.b.ii No withdrawals this year.

\section{PENNSYLVANIA STATE}

1.a

\section{QUEEN'S}

Volumes held June 30, 2009 revised to 45,721.

This figure represents titles where Hershey is the only owning library. The prior year figure of 23,741 included titles that were Hershey only, as well as joint purchases between Hershey and the UP Libraries. The decrease is due to this difference.

Number of consortial serial titles is unknown.

Reflects electronic media.

For the 2008-2009 ARL Survey, we estimated 93 linear $\mathrm{ft}$ in response to this question. The 2009-2010 figure of 1,129 linear $\mathrm{ft}$. is based upon physical measurement rather than an estimate.

Figure includes 242 videos and 129 multi-media materials.

The decrease in the number of presentations is due to a combination of the following: A key instructional position was unfilled for six months of the year resulting in a decrease in the total number of sessions; In 2009-2010 two new sessions were designed that presented asynchronous on-line course integrated instruction. Each of the new sessions was designed for a larger group of participants (145 participants per session). As a result, we noted an overall decrease in the number of sessions held but noted an overall increase in the number of participants.

In 2009-2010 two new sessions were designed that presented asynchronous on-line course integrated instruction. Each of the new sessions was designed for a larger group of participants (145 participants per session). As a result, we noted an overall decrease in the number of sessions held but noted an overall increase in the number of participants.

We are currently seeing a pattern of decreasing use.

All figures are as of $04 / 30 / 2010$. 
QUESTION FOOTNOTE

NUMBER

QUEEN'S, cont.

1.b, 1.b.ii Withdrawal of bound journals now available electronically.

16-26 Expenditures as reported in Canadian dollars: (16a) $\$ 127,608 ;(16 b) \$ 1,623,054 ;(16 c) \$ 0 ;(16 d) \$ 0 ;(16) \$ 1,750,662 ;(17) \$ 3,831 ;(18 a)$

$\$ 713,007$; (18b) \$471,773; (18c) \$33,761; (18) \$1,218,541; (20) \$171,421; (21) \$3,144,455; (22) \$0; (23) \$1,567,507; (24a) \$0; (24b) \$0; (25)

$\$ 0 ;(26) \$ 0$.

30, $31 \quad$ Fewer sessions offered, but class size increased.

\title{
ROCHESTER
}

1,5 Only print-only materials are reported here. River Campus Libraries is reporting e-materials for all UR libraries.

26 2008-2009 figure was overstated. Credits for document delivery and interlibrary lending were not deducted from costs prior to reporting.

\section{SASKATCHEWAN}

\author{
All figures are as of 04/30/2010. \\ 1.a \\ Volumes held June 30, 2009 revised to 164,020. \\ $16-26$ \\ Expenditures as reported in Canadian dollars: (16a) \$510,312; (16b) \$2,116,036; (16c) \$0; (16d) \$0; (16) \$2,626,348; (17) \$5,427; (18a) \\ $\$ 566,506$; (18b) \$265,987; (18c) \$31,440; (18) \$863,933; (20) \$0; (21) \$3,495,708; (22) \$0; (23) \$0; (24a) \$0; (24b) \$0; (25) \$0; (26) \$0.
}

\section{SOUTHERN CALIFORNIA}

Library branches included: Health Sciences Libraries - Medicine, Pharmacy and Dentistry.

Due to flat budget, fewer titles could be purchased.

5.a.ii

Publishers no longer provide free print with electronic subscriptions. Since our patrons prefer e-journals, print journals were canceled to fund other resources.

5.b More open access journals available in our subject fields.

16.c 2008-2009 amount was double counted; also a decrease in purchasing for 2009-2010 was due to a flat budget.

17 Includes repair and storage materials for archival gifts received in 2009-2010.

20 Reallocation of flat budget.

22 Decrease in print books and increase in e-book expenditures.

24.a Accounting issue resulted in under reporting in 2008-2009 and over reporting in 2009-2010.

30 Significantly expanded bioinformatics presentations and instructional consultations.

33, 34 Decrease due to more reliance on e-resources.

\section{TEMPLE}

Consolidated two locations into the new Ginsburg Health Sciences Library building.

\section{TENNESSEE}

Library branches included: Health Sciences Library-Memphis and Preston Medical Library-Knoxville.

\section{TEXAS TECH}

All figures are as of 08/31/2010.

Library branches included: Harrington Library TTUHSC-Amarillo; Montes-Gallo Library of the Health Sciences TTUHSC-El Paso; Library of the Health Sciences TTUHSC-Odessa; and the Preston Smith HSC Library.

\section{TULANE}


NuMBER

\section{VANDERBILT}

1.b

1.b.i

1.b.ii

3

5-5.a.ii

$16,16 . b$

16.a

16.c

18,27

18.a, 27.a

18.c

21

27.c

31

36

\section{VIRGINIA}

2

3

\section{WASHINGTON}

1.a Volumes held June 30, 2009 revised to 285,204.

\section{WASHINGTON U.-ST. LOUIS}

1.a

Volumes held June 30, 2009 revised to 294,975.

2

$5 . b$

For 2008-2009, the volume count was reported in error as a title count. The corrected number reported here reflects a title count.

YALE

1.b.i
Major weeding project resulted in large net decrease in collection size.

Increase to monographic budget for e-books.

Eskind Biomedical Library undertook a substantial weeding project during 2009-2010.

Increase in purchases due to increased monographic budget for e-books.

Move from print to electronic including multi-title databases. e.g. Micromedex, that are now paid through a separate Informatics Center budget.

Increased monographic budget to emphasize purchases of electronic content, e-books.

"Other" materials funds used for purchase of electronic journal backfiles.

Professionals providing non-traditional services are now paid from a separate budget under the Informatics Center.

Center. They are no longer paid staff of the Eskind Biomedical Library.

Reduction in student assistant funding due to less need for shelving with increased emphasis on electronic books and journals.

Total student hours are 30/week, i.e. .75 FTE.

Library emphasis on larger instruction groups which include resident and nursing student orientations.

Decreased need for ILL due to e-journal backfile purchases.

Includes 3,020 e-books.

Titles are reported under University Library for all branches.

Includes 550 e-books. 


\section{ARL Member Libraries as of January 1, 2011}

The Association of Research Libraries (ARL) represents the interests of 126 libraries that serve major research institutions in the US and Canada. The ARL Statistics and Measurement program is organized around identifying, collecting, analyzing, and distributing quantifiable information describing the characteristics of research libraries.

\begin{tabular}{|c|c|c|}
\hline Institution & Category & Full Name of Institution \\
\hline Alabama & $S$ & University of Alabama \\
\hline Alberta & $\mathrm{C}$ & University of Alberta \\
\hline Arizona & $S$ & University of Arizona \\
\hline Arizona State & $S$ & Arizona State University \\
\hline Auburn & S & Auburn University \\
\hline Boston & $\mathrm{P}$ & Boston University \\
\hline Boston College & $\mathrm{P}$ & Boston College \\
\hline Brigham Young & $\mathrm{P}$ & Brigham Young University \\
\hline British Columbia & $\mathrm{C}$ & University of British Columbia \\
\hline Brown & $\mathrm{P}$ & Brown University \\
\hline Berkeley, California & $S$ & University of California, Berkeley \\
\hline Calgary & $\mathrm{C}$ & University of Calgary \\
\hline California, Davis & $S$ & University of California, Davis \\
\hline California, Irvine & S & University of California, Irvine \\
\hline California, Los Angeles & S & University of California, Los Angeles \\
\hline California, Riverside & $S$ & University of California, Riverside \\
\hline California, San Diego & S & University of California, San Diego \\
\hline California, Santa Barbara & S & University of California, Santa Barbara \\
\hline Case Western Reserve & $\mathrm{P}$ & Case Western Reserve University \\
\hline Chicago & $\mathrm{P}$ & University of Chicago \\
\hline Cincinnati & S & University of Cincinnati \\
\hline Colorado & $S$ & University of Colorado \\
\hline Colorado State & $S$ & Colorado State University \\
\hline Columbia & $\mathrm{P}$ & Columbia University \\
\hline Connecticut & $\mathrm{S}$ & University of Connecticut \\
\hline Cornell & $\mathrm{P}$ & Cornell University \\
\hline Dartmouth & $\mathrm{P}$ & Dartmouth College \\
\hline Delaware & $S$ & University of Delaware \\
\hline Duke & $\mathrm{P}$ & Duke University \\
\hline Emory & $\mathrm{P}$ & Emory University \\
\hline Florida & S & University of Florida \\
\hline Florida State & $S$ & Florida State University \\
\hline George Washington & $\mathrm{P}$ & George Washington University \\
\hline Georgetown & $\mathrm{P}$ & Georgetown University \\
\hline Georgia & $\mathrm{S}$ & University of Georgia \\
\hline Georgia Tech & S & Georgia Institute of Technology \\
\hline Guelph & $\mathrm{C}$ & University of Guelph \\
\hline Harvard & $\mathrm{P}$ & Harvard University \\
\hline Hawaii & $S$ & University of Hawaii \\
\hline
\end{tabular}

\section{Location}

Tuscaloosa, Alabama

Edmonton, Alberta

Tucson, Arizona

Tempe, Arizona

Auburn, Alabama

Boston, Massachusetts

Boston, Massachusetts

Provo, Utah

Vancouver, British Columbia

Providence, Rhode Island

California, Berkeley

Calgary, Alberta

Davis, California

Irvine, California

Los Angeles, California

Riverside, California

La Jolla, California

Santa Barbara, California

Cleveland, Ohio

Chicago, Illinois

Cincinnati, Ohio

Boulder, Colorado

Fort Collins, Colorado

New York, New York

Storrs, Connecticut

Ithaca, New York

Hanover, New Hampshire

Newark, Delaware

Durham, North Carolina

Atlanta, Georgia

Gainesville, Florida

Tallahassee, Florida

Washington, DC

Washington, DC

Athens, Georgia

Atlanta, Georgia

Guelph, Ontario

Cambridge, Massachusetts

Honolulu, Hawaii 


\begin{tabular}{|c|c|c|}
\hline Institution & Category & Full Name of Institution \\
\hline Houston & S & University of Houston \\
\hline Howard & $\mathrm{P}$ & Howard University \\
\hline Illinois, Chicago & $S$ & University of Illinois at Chicago \\
\hline Illinois, Urbana & S & University of Illinois at Urbana \\
\hline Indiana & $S$ & Indiana University \\
\hline Iowa & $\mathrm{S}$ & University of Iowa \\
\hline Iowa State & S & Iowa State University \\
\hline Johns Hopkins & $\mathrm{P}$ & Johns Hopkins University \\
\hline Kansas & S & University of Kansas \\
\hline Kent State & $S$ & Kent State University \\
\hline Kentucky & $S$ & University of Kentucky \\
\hline Laval & $\mathrm{C}$ & Laval University \\
\hline Louisiana State & $S$ & Louisiana State University \\
\hline Louisville & $S$ & University of Louisville \\
\hline McGill & $\mathrm{C}$ & McGill University \\
\hline McMaster & $\mathrm{C}$ & McMaster University \\
\hline Manitoba & $\mathrm{C}$ & University of Manitoba \\
\hline Maryland & $S$ & University of Maryland \\
\hline Massachusetts & S & University of Massachusetts \\
\hline MIT & $\mathrm{P}$ & Massachusetts Institute of Technology \\
\hline Miami & $\mathrm{P}$ & University of Miami \\
\hline Michigan & S & University of Michigan \\
\hline Michigan State & $S$ & Michigan State University \\
\hline Minnesota & $S$ & University of Minnesota \\
\hline Missouri & S & University of Missouri \\
\hline Montreal & $\mathrm{C}$ & University of Montreal \\
\hline Nebraska & $S$ & University of Nebraska-Lincoln \\
\hline New Mexico & S & University of New Mexico \\
\hline New York & $\mathrm{P}$ & New York University \\
\hline North Carolina & $S$ & University of North Carolina \\
\hline North Carolina State & $S$ & North Carolina State University \\
\hline Northwestern & $\mathrm{P}$ & Northwestern University \\
\hline Notre Dame & $\mathrm{P}$ & University of Notre Dame \\
\hline Ohio & $S$ & Ohio University \\
\hline Ohio State & S & Ohio State University \\
\hline Oklahoma & $S$ & University of Oklahoma \\
\hline Oklahoma State & $S$ & Oklahoma State University \\
\hline Oregon & $S$ & University of Oregon \\
\hline Ottawa & $\mathrm{C}$ & University of Ottawa \\
\hline Pennsylvania & $\mathrm{P}$ & University of Pennsylvania \\
\hline Pennsylvania State & $S$ & Pennsylvania State University \\
\hline Pittsburgh & $S$ & University of Pittsburgh \\
\hline Princeton & $\mathrm{P}$ & Princeton University \\
\hline Purdue & $S$ & Purdue University \\
\hline
\end{tabular}

\section{Location}

Houston, Texas

Washington, DC

Chicago, Illinois

Urbana, Illinois

Bloomington, Indiana

Iowa City, Iowa

Ames, Iowa

Baltimore, Maryland

Lawrence, Kansas

Kent, Ohio

Lexington, Kentucky

Quebec, Quebec

Baton Rouge, Louisiana

Louisville, Kentucky

Montreal, Quebec

Hamilton, Ontario

Winnipeg, Manitoba

College Park, Maryland

Amherst, Massachusetts

Cambridge, Massachusetts

Coral Gables, Florida

Ann Arbor, Michigan

East Lansing, Michigan

Minneapolis, Minnesota

Columbia, Missouri

Montreal, Quebec

Lincoln, Nebraska

Albuquerque, New Mexico

New York, New York

Chapel Hill, North Carolina

Raleigh, North Carolina

Evanston, Illinois

Notre Dame, Indiana

Athens, Ohio

Columbus, Ohio

Norman, Oklahoma

Stillwater, Oklahoma

Eugene, Oregon

Ottawa, Ontario

Philadelphia, Pennsylvania

University Park, Pennsylvania

Pittsburgh, Pennsylvania

Princeton, New Jersey

West Lafayette, Indiana 


\section{Institution}

Queen's

Rice

Rochester

Rutgers

Saskatchewan

South Carolina

Southern California

Southern Illinois

SUNY-Albany

SUNY-Buffalo

SUNY-Stony Brook

Syracuse

Temple

Tennessee

Texas

Texas A\&M

Texas Tech

Toronto

Tulane

Utah

Vanderbilt

Virginia

Virginia Tech

Washington

Washington State

Washington U.-St. Louis

Waterloo

Wayne State

Western Ontario

Wisconsin

Yale

York

Boston Public Library

Canada Inst. SciTech Info.

Center for Research Libraries

Library and Archives Canada

Library of Congress

National Agricultural Library

National Archives \& Records

National. Library of Medicine

New York Public Library

New York State Library

Smithsonian Institution

\section{Category Full Name of Institution}

C Queen's University

P Rice University

P University of Rochester

S Rutgers University

C University of Saskatchewan

S University of South Carolina

P University of Southern California

S Southern Illinois University

S University at Albany, State University of New York

S University at Buffalo, State University of New York

S State University of New York at Stony Brook

P Syracuse University

S Temple University

S University of Tennessee

S University of Texas

S Texas A\&M University

S Texas Tech University

C University of Toronto

P Tulane University

S University of Utah

P Vanderbilt University

S University of Virginia

S Virginia Polytechnic Institute \& State University

$S \quad$ University of Washington

S Washington State University

P Washington University

C University of Waterloo

S Wayne State University

C University of Western Ontario

$\mathrm{S} \quad$ University of Wisconsin

P Yale University

C York University

N Boston Public Library

X Canada Inst. for Scientific \& Technical Information

N Center for Research Libraries

X Library and Archives Canada

$\mathrm{N} \quad$ Library of Congress

N National Agricultural Library

N National Archives \& Records Administration

N National Library of Medicine

N New York Public Library

N New York State Library

N Smithsonian Institution

\section{Location}

Kingston, Ontario

Houston, Texas

Rochester, New York

New Brunswick, New Jersey

Saskatoon, Saskatchewan

Columbia, South Carolina

Los Angeles, California

Carbondale, Illinois

Albany, New York

Buffalo, New York

Stony Brook, New York

Syracuse, New York

Philadelphia, Pennsylvania

Knoxville, Tennessee

Austin, Texas

College Station, Texas

Lubbock, Texas

Toronto, Ontario

New Orleans, Louisiana

Salt Lake City, Utah

Nashville, Tennessee

Charlottesville, Virginia

Blacksburg, Virginia

Seattle, Washington

Pullman, Washington

St. Louis, Missouri

Waterloo, Ontario

Detroit, Michigan

London, Ontario

Madison, Wisconsin

New Haven, Connecticut

North York, Ontario

Boston, Massachusetts

Ottawa, Ontario

Chicago, Illinois

Ottawa, Ontario

Washington, DC

Beltsville, Maryland

Washington, DC

Bethesda, Maryland

New York, New York

Albany, New York

Washington, DC 
S: US public university $P$ : US private university $\mathrm{C}$ : Canadian university $\mathrm{N}$ : US nonuniversity

$\mathrm{X}$ : Canadian nonuniversity 
